\title{
Fröhlich polaron effective mass and localization length in cubic materials: Degenerate and anisotropic electronic bands
}

\author{
Bogdan Guster $\odot,{ }^{1}$ Pedro Melo ${ }^{\circ},{ }^{2}$ Bradley A. A. Martin ${ }^{\circ},{ }^{3}$ Véronique Brousseau-Couture ${ }^{\circ},{ }^{4}$ Joao C. de Abreu, ${ }^{2}$ \\ Anna Miglio, ${ }^{1}$ Matteo Giantomassi $\odot{ }^{1}{ }^{1}$ Michel Côté $\odot,{ }^{5}$ Jarvist M. Frost, ${ }^{3}$ Matthieu J. Verstraete, ${ }^{2}$ and Xavier Gonze ${ }^{1,6}$ \\ ${ }^{1}$ European Theoretical Spectroscopy Facility, Institute of Condensed Matter and Nanosciences, Université Catholique de Louvain, \\ Chemin des étoiles 8, bte L07.03.01, B-1348 Louvain-la-Neuve, Belgium \\ ${ }^{2}$ NanoMat/Q-Mat/CESAM and European Theoretical Spectroscopy Facility, Université de Liège (B5), B-4000 Liège, Belgium \\ ${ }^{3}$ Department of Physics, Imperial College London, Exhibition Road, London SW7 2AZ, England, United Kingdom \\ ${ }^{4}$ Département de Physique et Regroupement Québécois sur les Matériaux de Pointe, Université de Montreal, \\ C.P. 6128, Succursale Centre-Ville, Montreal, Canada H3C $3 J 7$ \\ ${ }^{5}$ Département de Physique, Université de Montreal, C.P. 6128, Succursale Centre-Ville, Montreal, Canada H3C 3J7 \\ ${ }^{6}$ Skolkovo Institute of Science and Technology, Skolkovo Innovation Center, Nobel St. 3, Moscow 143026, Russia
}

(Received 28 September 2021; accepted 22 November 2021; published 13 December 2021)

\begin{abstract}
Polarons, that is, charge carriers correlated with lattice deformations, are ubiquitous quasiparticles in semiconductors, and play an important role in electrical conductivity. To date most theoretical studies of so-called large polarons, in which the lattice can be considered as a continuum, have focused on the original Fröhlich model: a simple (nondegenerate) parabolic isotropic electronic band coupled to one dispersionless longitudinal optical phonon branch. The Fröhlich model allows one to understand characteristics such as polaron formation energy, radius, effective mass, and mobility. Real cubic materials, instead, have electronic band extrema that are often degenerate (e.g., threefold degeneracy of the valence band), or anisotropic (e.g., conduction bands at $X$ or $L$ ), and present several phonon modes. In the present paper, we address such issues. We keep the continuum hypothesis inherent to the large polaron Fröhlich model, but waive the isotropic and nondegeneracy hypotheses, and also include multiple phonon branches. For polaron effective masses, working at the lowest order of perturbation theory, we provide analytical results for the case of anisotropic electronic energy dispersion, with two distinct effective masses (uniaxial) and numerical simulations for the degenerate three-band case, typical of III-V and II-VI semiconductor valence bands. We also deal with the strong-coupling limit, using a variational treatment: we propose trial wave functions for the above-mentioned cases, providing polaron radii and energies. Then, we evaluate the polaron formation energies, effective masses, and localization lengths using parameters representative of a dozen II-VI, III-V, and oxide semiconductors, for both electron and hole polarons. We show that for some cases perturbation theory (the weak-coupling approach) breaks down. In some other cases, the strong-coupling approach reveals that the large polaron hypothesis is not valid, which is another distinct breakdown. In the nondegenerate case, we compare the perturbative approach with the Feynman path integral approach in characterizing polarons in the weak-coupling limit. Thus, based on theoretical results for cubic materials, the present paper characterizes the validity of the continuum hypothesis for a large set of 20 materials.
\end{abstract}

DOI: 10.1103/PhysRevB.104.235123

\section{INTRODUCTION}

Polarons are usually defined as a correlated state combining a charge carrier and a deformation of the lattice. They are ubiquitous quasiparticles arising naturally in many insulators and semiconductors [1]. Although the polaron concept can be traced back to work by Landau [2] in the 1930s, the polaron term was introduced later by Pekar [3]. Early models assumed the localization length of the polaron to be much larger than the crystal atomic periodicity (large polaron), so that the charged particle couples with the polarization of the lattice through a macroscopically screened Coulomb interaction. A formalism for determining large polaron properties based on a strong-coupling approach was provided first by Landau and
Pekar [4]. Later, Fröhlich [5] introduced a second-quantized formulation, and worked out a weak-coupling approach to large polarons. The Fröhlich model can be studied also by more sophisticated formalisms, such as Feynman's path integral variational approach [6] or diagrammatic quantum Monte Carlo [7], valid for the whole range of coupling strengths. Such approaches target polaron formation energy, effective mass, mobility, and optical characteristics [8]. Alternatively, the Holstein model has been a successful theoretical tool for studying small polarons, in which the discrete character of the lattice is retained, and the electron-phonon interaction is short-ranged $[9,10]$.

The full complexity of materials is however not captured by these models. Most real materials have several phonon 
branches and electronic bands, both with arbitrary dispersions (albeit quadratic close to the extrema), and general electron-phonon interaction, though it generically reduces to the Fröhlich form for small momentum transfer. Such features can only be addressed by first-principles approaches, that have seen an impressive development during the last two decades. Existing first-principles approaches consider polarons either in the self-trapped adiabatic state [1,11-15], corresponding to the strong-coupling limit, or from many-body perturbation theory, corresponding to the weak-coupling limit, e.g., the Allen-Heine-Cardona (AHC) approach [16-27]. In the first case, the polaron formation energy is computed from the associated collective atomic displacement pattern and frozen electronic density, and the mobility is estimated through the computation of barriers for transitioning the localized polaron from one site to another. In the second case, the treatment of the correlation between electron and phonon delivers a modification of the band edge eigenenergies, and a modification of the band gap often termed zero-point renormalization (ZPR), and also allows one to compute spectral functions. Only recently a path towards a unified first-principles theory of polarons has been sketched [25], and will likely be followed by further developments.

In Ref. [26] Miglio et al. established the predictive power of the first-principles approach for the ZPR, in the AHC methodology, by studying a set of 30 materials, and performed systematic comparison with existing experimental data. The inclusion of nonadiabatic effects was demonstrated to be essential to produce a match between experimental and theoretical ZPR of the band gap. This was made clear by introducing a generalized Fröhlich model $(\mathrm{gFr})$ from which the ZPR could be deduced without resorting to full first-principles calculations, as only a few macroscopic quantities, determined either from experiment or first-principles calculations, were needed. This generalized Fröhlich model will be at the center of the present paper.

Indeed, the continuum hypothesis inherent to the Fröhlich model can be dissociated from further simplifications present in the original Fröhlich model. These additional simplifications are (i) only one isotropic LO phonon branch, (ii) only one isotropic nondegenerate electronic band, and (iii) an isotropic dielectric tensor to determine the electronphonon coupling (EPC). One can waive such restrictions without addressing the full complexity of the materials, in particular without introducing full-fledged electronic and phononic dispersions in the full Brillouin zone. By considering parabolic bands and dispersionless phonon branches, still allowing anisotropic behaviors and including nondegeneracy and warping effects [28], one captures an important part of the electron-phonon effect in real materials, so that such generalized Fröhlich model is sufficiently predictive for the most ionic materials among those considered, namely, for the oxides and II-VI materials. Miglio et al. provided the polaron formation energy within the lowest-order perturbation theory for the generalized Fröhlich model. For each of the examined materials, less than a dozen parameters defined the gFr model, depending on the symmetries and number of atoms per cell.

The original Fröhlich model, that is characterized by one single adimensional parameter, $\alpha$, has been studied by many different theoretical approaches. These studies determined the ground-state energy as a function of $\alpha$, but also the polaron effective mass, mobility, optical properties, localization, or modifications due to magnetic field, be they in three-dimensional or confined situations [29-31]. The polaron effective mass, determined in the weak-coupling regime at the lowest order of perturbation, diverges when $\alpha$ is 6 or bigger, indicating the breakdown of perturbation theory. The localization radius, estimated from the strong-coupling limit, provides a criterion to determine whether the large polaron picture is correct: if it is comparable to or only slightly bigger than the distance between equivalent sites, the large polaron hypothesis breaks down.

So far, there have only been a few attempts to formulate and study an extended model beyond the above-mentioned simplifying hypotheses. Trebin and Rössler [32] studied polaron energies and effective masses in the case of triply degenerate bands, however without the inclusion of band warping (they worked with "isotropic" triply degenerate bands), and ignored the effect of multiple LO phonon branches. Similarly, Fock, Kramer, and Büttner [33] examined polaron energies and masses in the nondegenerate case with uniaxial symmetry for effective mass (and dielectric tensor), but considered only one LO phonon branch. The case of multiple phonon branches was tackled by Schlipf, Poncé, and Giustino [34], although they focused on the nondegenerate case with isotropic effective masses. Exact formulas were derived in these studies, while others [35,36] proposed approximate results. To our knowledge, the strong-coupling limit has not been examined in any of these cases. Trebin and Rössler [32] also considered the effect of spin-orbit coupling. It is not considered in the present paper, and must be the subject of future work. A supplementary issue that is not considered in the present paper has been recently tackled by Houtput and Tempere [37] in deriving anharmonic contributions to the Fröhlich model.

In order to study polarons in more realistic cases, and pursue the work of Miglio et al., who focused on the polaron energies only, we compute effective masses in the weakcoupling limit, and further estimate localization lengths in the strong-coupling limit. We limit our investigation of the generalized Fröhlich model to the case of cubic materials, treated in the harmonic approximation for phonons, and in the same macroscopic continuum hypothesis as in the usual Fröhlich model. We establish criteria for the breakdown of perturbation theory, similar to that for a single isotropic nondegenerate electronic band, and obtain an estimation of the different (anisotropic) localization lengths, to be compared with the interatomic distance.

In Sec. II we summarize the basic theoretical knowledge of large polarons properties. First, we consider results for the standard Fröhlich model, in the weak- and strong-coupling limits, then present the Feynman variational approach, valid for the whole coupling range. We report the polaron formation energy from the gFr model, specialized for the cubic case, for isotropic and anisotropic and for nondegenerate and degenerate electronic dispersions. In Sec. III we derive the polaron effective mass for nondegenerate anisotropic as well as degenerate electronic bands coupled to multiple phonon modes. In Sec. IV we provide the localization lengths beyond the basic Fröhlich model, for the same scenarios as for the polaron effective masses. In Sec. V, we present our results 
(effective masses and localization lengths) for a subset of 21 cubic materials among those originally considered by Miglio et al. In the discussion Sec. VI, we combine the effective masses and localization length data, to assess, for each material and for both electron and hole polarons, whether there is a breakdown of perturbation theory and whether the continuum hypothesis is valid or not. Such breakdowns happen indeed for several oxide materials. We also compare, for the nondegenerate uniaxial case, our formation energies and effective masses with those from the Feynman path integral approach for the isotropic case with effective masses equal to those of the uniaxial case, approximately averaged. We conclude in Sec. VII.

\section{THEORETICAL BACKGROUND}

\section{A. Polaron energy, effective mass, and localization radius in the Fröhlich model}

The basic Fröhlich model [38] starts from one electronic band with spherical symmetry (i.e., isotropic), with parabolic dispersion determined by the effective mass $m^{*}$, coupled to one dispersionless longitudinal optical phonon of frequency $\omega_{\mathrm{LO}}$ while ignoring the crystal discrete nature (continuum limit). The Fröhlich model is thus able to describe a very reduced set of realistic scenarios, where e.g., compounds are binary, the band extrema are neither anisotropic nor degenerate, and one ignores the contribution to the polaron formation due to other phonon modes (e.g., acoustic and transverse optical).

Let us first recall the Fröhlich Hamiltonian describing this picture (everything will be expressed in atomic units unless stated otherwise: $m_{e}=1,|e|=1, \hbar=1$ ):

$$
\hat{H}^{\mathrm{Fr}}=\hat{H}_{e}^{\mathrm{Fr}}+\hat{H}_{p}^{\mathrm{Fr}}+\hat{H}_{\mathrm{EPC}}^{\mathrm{Fr}},
$$

where

$$
\begin{gathered}
\hat{H}_{e}^{\mathrm{Fr}}=\sum_{\vec{k}} \frac{\vec{k}^{2}}{2 m^{*}} \hat{c}_{\vec{k}}^{+} \hat{c}_{\vec{k}}, \\
\hat{H}_{p}^{\mathrm{Fr}}=\sum_{\vec{q}} \omega_{\mathrm{LO}} \hat{a}_{\vec{q}}^{+} \hat{a}_{\vec{q}}, \\
\hat{H}_{\mathrm{EPC}}^{\mathrm{Fr}}=\sum_{\vec{k}, \vec{q}} g^{\mathrm{Fr}}(\vec{q}) \hat{c}_{\vec{k}}^{+} \hat{c}_{\vec{k}}\left(\hat{a}_{\vec{q}}+\hat{a}_{-\vec{q}}^{+}\right),
\end{gathered}
$$

where $\hat{c}_{\vec{k}}^{+}\left(\hat{c}_{\vec{k}}\right)$ is the electron creation (annihilation) operator and $\hat{a}_{\vec{q}}^{+}\left(\hat{a}_{\vec{q}}\right)$ is the phonon creation (annihilation) operator. The electron-phonon coupling is defined as follows:

$$
g^{\mathrm{Fr}}(\vec{q})=\frac{i}{q}\left[\frac{2 \pi \omega_{\mathrm{LO}}}{V_{\mathrm{BvK}}}\left(\frac{1}{\epsilon^{\infty}}-\frac{1}{\epsilon^{0}}\right)\right]^{1 / 2},
$$

where $q$ is the norm of the phonon wave vector $\vec{q}$ and $V_{\mathrm{BvK}}$ is the Born-von Kármán volume. This, in turn, can be rewritten as

$$
g^{\mathrm{Fr}}(\vec{q})=\frac{i}{q}\left[\frac{2 \sqrt{2} \pi}{V_{\mathrm{BvK}}} \frac{\omega_{\mathrm{LO}}^{3 / 2}}{\sqrt{m^{*}}} \alpha\right]^{1 / 2},
$$

with the adimensional parameter $\alpha$

$$
\alpha=\left(\epsilon^{*}\right)^{-1}\left(m^{*}\right)^{1 / 2}\left(2 \omega_{\mathrm{LO}}\right)^{-1 / 2},
$$

where

$$
\left(\epsilon^{*}\right)^{-1}=\left(\epsilon^{\infty}\right)^{-1}-\left(\epsilon^{0}\right)^{-1} .
$$

The treatment of the Fröhlich Hamiltonian at the lowest order of perturbation theory in $\alpha$ (second-order perturbation theory in the strength of the electron-phonon coupling) gives the polaron binding energy as a function of the crystalline momentum:

$$
E_{P}(\vec{k})=\frac{k^{2}}{2 m^{*}}-\alpha \frac{\omega_{\mathrm{LO}}}{k a_{\mathrm{LO}}} \arcsin \left(k a_{\mathrm{LO}}\right),
$$

where we define a characteristic length [39]:

$$
a_{\mathrm{LO}}=\left(2 m^{*} \omega_{\mathrm{LO}}\right)^{1 / 2} .
$$

Expanding the arcsin in a series up to the second-order term in $k a_{\mathrm{LO}}$ one obtains the (isotropic) polaronic dispersion relation:

$$
E_{P}(\vec{k})=-\alpha \omega_{\mathrm{LO}}+\frac{k^{2}}{2 m^{*}}\left(1-\frac{\alpha}{6}\right)+\mathcal{O}\left(k^{4}\right),
$$

with the zero momentum value also known as the ZPR.

From the previous expression the polaron effective mass expression in the lowest-order perturbation theory is

$$
m_{P}^{*}=m^{*}\left(1-\frac{\alpha}{6}\right)^{-1} .
$$

This polaron effective mass expression changes sign for $\alpha \geqslant 6$, indicating the breakdown of perturbation theory. Even so, it is important to note that for values of the coupling constant close to 6 the polaron formation energy in the lowest-order perturbation theory treatment shows only mild deviations $(\approx 10-15 \%)$ with respect to the diagrammatic Monte Carlo treatment [7]. Considering that we are dealing with lowestorder perturbation theory, the breakdown limit can be taken as a qualitative one, which signals a crossing from the weak- to a strong-coupling regime in the Fröhlich picture. In this latter scenario the polaron reaches a self-trapping regime.

So far we have discussed the energetics of the polaron and effective mass. We now tackle another important characteristic of a polaron, namely, its localization length. In the simplest case where the electronic band is isotropic, the theory describing the polaron localization has been developed by Pekar [3] and Landau and Pekar [4] in the strong-coupling theory framework, using a variational approach based on a Gaussian wave-function ansatz. This method is described extensively by Mahan (see Ref. [40], Sec. 7.1.3). The approach is based on the variational principle, whereby the energy associated to a normalized trial wave function $\phi(\vec{r})$ combines an electronic kinetic energy part and a coupled electron-phonon and phonon part. Explicitly,

$$
E_{P}[\phi(\vec{r})]=E_{\mathrm{el}}[\phi(\vec{r})]+E_{e p p}[\phi(\vec{r})],
$$

where the electronic energy is written

$$
E_{\mathrm{el}}[\phi(\vec{r})]=\int d \vec{r} \frac{|\vec{\nabla} \phi(\vec{r})|^{2}}{2 m^{*}},
$$

while the electronic density,

$$
\rho(\vec{r})=|\phi(\vec{r})|^{2},
$$


induces a response of the polarizable medium, obtained mathematically by considering its Fourier transform

$$
\rho(\vec{k})=\frac{1}{(2 \pi)^{3 / 2}} \int d \vec{r} \rho(\vec{r}) e^{i \vec{k} \vec{r}}
$$

and building the associated electron-phonon and phonon energy:

$$
E_{e p p}[\phi(\vec{r})]=-\int d \vec{k} \frac{4 \pi}{2 k^{2} \epsilon^{*}} \rho^{*}(\vec{k}) \rho(\vec{k}) .
$$

The electron wave function is frozen, as well as the deformation of the polarizable medium. Hence, in this strongcoupling scenario, one works in an adiabatic approximation, in which the electron and phonon parts are explicitly correlated, albeit frozen. A discussion of the leftover part is also provided in Ref. [40], showing how the variational approach was obtained from the full second-quantized Hamiltonian, to which both weak- and strong-coupling approaches can be applied, as well as the other methodologies mentioned in the introduction.

Different simple trial wave functions can be found in the literature, giving analytical results. The convenient Gaussian trial wave function [40] is written

$$
\phi(\vec{r})=\left(\frac{1}{\sqrt{\pi} a_{P}}\right)^{3 / 2} \exp \left(-\frac{r^{2}}{2 a_{P}^{2}}\right),
$$

where $a_{P}$ is the localization radius of the electron, an adjustable parameter in the polaron wave function, allowing the minimization of the polaron formation energy.

The best (in the variational sense) localization length is then obtained in terms of the electron effective mass $m^{*}$ and the dielectric constant $\epsilon^{*}$, defined in Eq. (8):

$$
a_{P}=\frac{3}{2} \sqrt{2 \pi}\left(m^{*}\right)^{-1} \epsilon^{*} .
$$

This adiabatic localization length is independent from the phonon frequency, and is roughly 17\% larger than the polaron localization length derived using an exponential wave-function ansatz [see Sio et al. [25], Eq. (11)]. The polaron localization can be expressed in terms of the phonon energy if one reintroduces the $\alpha$ parameter:

$$
a_{P}=\frac{3}{2} \sqrt{\pi}\left(m^{*} \omega_{\mathrm{LO}}\right)^{-1 / 2}(\alpha)^{-1} .
$$

The localization length is not a hard limit on the polaron extent, but indicates a characteristic decay length of the selftrapped electronic wave function.

Along the same lines one can provide the polaron groundstate energy in the spherical limit within the Gaussian variational strong-coupling approach:

$$
E_{P}=-\frac{1}{6 \pi} m^{*}\left(\epsilon^{*-1}\right)^{2} .
$$

This Gaussian polaron stabilization energy is roughly $8 \%$ lower than with the exponential ansatz [see Sio et al. [25], Eq. (12)], suggesting a Gaussian wave-function ansatz seems to be a superior choice to start with in the variational approach.

Rewriting the previous equation in terms of the coupling constant $\alpha$, one arrives at

$$
E_{P}=-\frac{1}{3 \pi} \omega_{\mathrm{LO}} \alpha^{2} \approx-\omega_{\mathrm{LO}}\left(0.1061 \alpha^{2}\right) .
$$

A much more sophisticated attempt at determining the polaron ground-state energy in the strong-coupling limit has been provided by Miyake [41], which shows a slightly different prefactor of the term quadratic in the coupling constant $\alpha$, and adds a constant term, in a perturbation expansion with small parameter $1 / \alpha^{2}$ :

$$
E_{P} \approx-\omega_{\mathrm{LO}}\left[0.1085 \alpha^{2}+2.836+\mathcal{O}\left(1 / \alpha^{2}\right)\right] .
$$

In the weak-coupling regime the polaron ground-state energy shows a leading linear dependence on $\alpha$, while in the strong-coupling regime a quadratic one. The crossing between the two different regimes, weak and strong, given by Eqs. (11) and (22) gives a value of $\alpha \approx 9.5$ (leaving aside the constant shift in the strong-coupling expansion).

\section{B. Polaron energy, effective mass, and radius in the Feynman approach}

The Feynman [6] 1955 path integral approach to the Fröhlich model casts the Fröhlich Hamiltonian [Eq. (1)] into a Lagrangian by passing the electron and phonon creation and annihilation operators into the corresponding coordinates and momenta, and performing a Legendre transformation. After a Gaussian integration over the momenta, one is left with a configuration path integral that is Gaussian over the phonon coordinates which can be evaluated directly. This results in an effective action of an electron coupled by a nonlocal Coulomb potential to a second fictitious particle:

$$
Z=\operatorname{Tr} \exp \left(-\beta \hat{H}^{\mathrm{Fr}}\right) \approx \int_{\vec{r}(0)=\vec{r}^{\prime}(\beta)} \mathcal{D} \vec{r}(\tau) e^{-S[\vec{r}(\tau)]},
$$

with

$$
\begin{aligned}
S[\vec{r}(\tau)]= & \frac{m^{*}}{2} \int_{0}^{\beta} d \tau\left(\frac{d \vec{r}(\tau)}{d \tau}\right)^{2}-\frac{\left(\omega_{\mathrm{LO}}\right)^{3 / 2} \alpha}{\sqrt{8 m^{*}}} \\
& \times \int_{0}^{\beta} d \tau \int_{0}^{\beta} d \sigma|\vec{r}(\tau)-\vec{r}(\sigma)|^{-1} e^{-\omega_{\mathrm{LO}}|\tau-\sigma|}
\end{aligned}
$$

Here $S$ is the (Fröhlich ) model action, and $Z$ is the partition function for the electron to begin and end at the same position over imaginary time $i \hbar \beta$, with the thermodynamic beta $\beta=$ $1 / k_{B} T$. This form of $S$ already assumes that $\beta$ is large (low temperature, relative to the phonon energy).

The path integral is difficult to evaluate for the $1 / r$ Coulomb potential, so Jensen's inequality, $\langle\exp f\rangle \geqslant \exp \langle f\rangle$, is used to approximate the model action $S$ by an analytically path-integrable quadratic action (the trial action), $S_{0}$, that has a time-retarded potential and a (tunable) exponential factor similar to $S$ :

$$
\begin{aligned}
S_{0}[\vec{r}(\tau)]= & \frac{m^{*}}{2} \int_{0}^{\beta} d \tau\left(\frac{d \vec{r}(\tau)}{d \tau}\right)^{2} \\
& +\frac{C}{2} \int_{0}^{\beta} d \tau \int_{0}^{\beta} d \sigma[\vec{r}(\tau)-\vec{r}(\sigma)]^{2} e^{-w|\tau-\sigma|} .
\end{aligned}
$$

This results in the Feynman-Jensen inequality that gives a solvable upper bound to the model free energy,

$$
F \leqslant F_{S_{0}}+\frac{1}{\beta}\left\langle S-S_{0}\right\rangle_{S_{0}},
$$


where $F_{S_{0}}$ is the free energy of the trial system and $\left\langle S-S_{0}\right\rangle_{S_{0}}$ is the expectation value of the difference in the two actions, taken with respect to the trial system:

$$
\left\langle S-S_{0}\right\rangle_{S_{0}}=\frac{\int \mathcal{D} \vec{r}(\tau)\left(S-S_{0}\right) e^{-S_{0}[\vec{r}]}}{\int \mathcal{D} \vec{r}(\tau) e^{-S_{0}[\vec{r}]}} .
$$

The ground-state energy $E$ is estimated by taking the limit $\beta \rightarrow \infty$ (temperature goes to zero). In this limit the trace in Eq. (24) can be estimated by the ground state, $\left.\operatorname{Tr} \exp (-\beta \hat{H})\right|_{\beta \rightarrow \infty} \approx \exp (-\beta E)$. The Feynman-Jensen inequality for the ground-state energy is thus

$$
\begin{aligned}
E & \leqslant \frac{3 \omega_{\mathrm{LO}}}{4 v}(v-w)^{2}-\frac{\alpha \omega_{\mathrm{LO}}}{\sqrt{\pi}} \frac{v}{w} \int_{0}^{\infty} d \tau \frac{e^{-\tau}}{\sqrt{\tau D(\tau)}}, \\
D(\tau) & =1+\frac{v\left(1-w^{2} / v^{2}\right)\left(1-e^{-v \tau}\right)}{\tau w^{2}},
\end{aligned}
$$

where $v^{2} \equiv w^{2}+4 C / w$. The process is variational with two free parameters: $v$ (the frequency of the harmonic oscillator composed of the electron and the fictitious particle) and $w$ (the exponential rate of decay of the coupling in time).

For extremal values of the coupling $\alpha, w$ smoothly approaches limits of 1 and 3. In the weak-coupling (small alpha) limit the energy minimum occurs when $v$ is near $w$. Therefore, Feynman set $v=(1+\varepsilon) w$ where $\varepsilon$ is small and expanded the energy expression [right-hand side of Eq. (29)] with respect to $\varepsilon$. Feynman then minimized the energy first with respect to $\varepsilon$ and then with respect to $w$ and found that in the weakcoupling limit the energy is least when

$$
\begin{aligned}
\frac{w}{\omega_{\mathrm{LO}}} & =3, \quad \frac{v}{\omega_{\mathrm{LO}}}=3\left[1+\frac{2 \alpha}{3 w}\left(1-\frac{2}{w}[\sqrt{w-1}-1]\right)\right] \\
\frac{E}{\omega_{\mathrm{LO}}} & \leqslant-\alpha-1.23\left(\frac{\alpha}{10}\right)^{2} .
\end{aligned}
$$

In the strong-coupling (large alpha) limit $v$ is large and $w$ approaches 1 so $w / v<<1$. Therefore, Feynman expanded the energy expression with respect to $w / v$ and then minimized the energy with respect to $v$ and $w$ and found that in the strong-coupling limit the energy is least when

$$
\begin{gathered}
\frac{w}{\omega_{\mathrm{LO}}}=1, \quad \frac{v}{\omega_{\mathrm{LO}}}=\frac{4 \alpha^{2}}{9 \pi}-4\left(\log 2+\frac{1}{2} \gamma\right)+1, \\
\frac{E}{\omega_{\mathrm{LO}}} \leqslant-\frac{\alpha^{2}}{2 \pi}-\frac{3}{2}(2 \log 2+\gamma)-\frac{3}{4}+\mathcal{O}\left(\frac{1}{\alpha^{2}}\right),
\end{gathered}
$$

where $\gamma=0.5772 \ldots$ is the Euler-Mascheroni constant.

For finite temperature a numeric variational solution is required. As temperature is relative to the phonon energy, soft polar materials will require a numeric solution at room temperature. The variational parameters for finite temperature can be evaluated by numerically minimizing Ōsaka's finite temperature generalization of Feynman's variational principle [42] to give a lower upper bound to the polaron free energy.

The effective polaron mass at zero temperature was found by Feynman [6] by assuming that the electron moves with a small velocity $\vec{u}$ from an initial coordinate $\overrightarrow{0}$ to a final coordinate $\vec{r}_{f}=\vec{u} \hbar \beta$ in an imaginary time $\hbar \beta$. Feynman then sought the total energy of the polaron and equated it to the form $E_{0}+\frac{1}{2} m_{P}^{*} u^{2}$ by expanding the total energy expression to quadratic order in the velocity $\vec{u}$. From the kinetic energy term Feynman found the polaron effective mass:

$$
m_{P}^{* F}=m^{*}\left[1+\frac{\alpha}{3 \sqrt{\pi}}\left(\frac{v}{w}\right)^{3} \int_{0}^{\infty} d \tau \frac{e^{-\tau} \tau^{1 / 2}}{[D(\tau)]^{3 / 2}}\right]
$$

where the values of the variational parameters are those that minimize the original polaron ground-state energy when $u=0$ in Eq. (29). From the values in Eq. (30) Feynman obtained the weak-coupling expression

$$
m_{P}^{* F}=m^{*}\left[1+\frac{1}{6} \alpha+0.025 \alpha^{2}+\cdots\right],
$$

and from the values in (31) the strong-coupling expression is

$$
m_{P}^{* F}=m^{*} \frac{160}{81}\left(\frac{\alpha}{\pi}\right)^{4} .
$$

At finite temperatures the effective polaron mass is proportional to the imaginary part of the complex impedance function $Z(\nu, \beta)$ provided by Feynman et al. [43] [Eqs. (35), (36), and (41)] in the zero frequency limit $v \rightarrow 0$ :

$$
m_{P}^{* F}(\beta)=m^{*} \lim _{v \rightarrow 0}\left\{\frac{\operatorname{Im} Z(\nu, \beta)}{v}\right\}
$$

as described by Peeters and Devreese [44].

Schultz [45] estimated the polaron size by calculating the root mean square distance between the electron and the fictitious particle. The reduced mass of their relative motion is

$$
\mu=\frac{m^{*}}{m_{P}^{* F}}\left(m_{P}^{* F}-m^{*}\right) .
$$

Schultz then used the ground-state harmonic oscillator wave function for the relative coordinate $\rho$,

$$
\phi_{0}(\rho)=\left(\frac{\mu v \omega_{\mathrm{LO}}}{\pi}\right)^{3 / 4} \exp \left(-\frac{\mu v \omega_{\mathrm{LO}} \rho^{2}}{2}\right),
$$

to define a polaron radius:

$$
r_{P} \equiv\left\langle\rho^{2}\right\rangle^{1 / 2}=\frac{1}{2}\left(\frac{3}{\mu v \omega_{\mathrm{LO}}}\right)^{1 / 2} .
$$

In the weak-coupling limit this is

$$
r_{P} \sim \frac{3}{4}\left(\frac{6}{\alpha m^{*} \omega_{\mathrm{LO}}}\right)^{1 / 2},
$$

and in the strong-coupling limit

$$
r_{P} \sim \frac{3}{2 \alpha}\left(\frac{\pi}{m^{*} \omega_{\mathrm{LO}}}\right)^{1 / 2} .
$$

In the degenerate anisotropic uniaxial case, we propose to naïvely incorporate the anisotropy into the Feynman approach (which is one-dimensional due to the underlying isotropy of the Fröhlich Hamiltonian) by treating the two directions independently with effective masses $m_{\perp}$ and $m_{z}$. We then use the variational principle separately in each direction to find the variational parameters $v_{\perp / z}$ and $w_{\perp / z}$ that give the lowest upper bound to the ground-state energy $E_{\perp / z}$ for each direction. The variational parameters can then be used to obtain the effective polaron masses $m_{P, \perp}^{* F}$ and $m_{P, z}^{* F}$ using Eq. (32) and polaron radii $r_{P \perp}$ and $r_{P z}$ using Eq. (38). 
We define an effective ground-state energy by taking the arithmetic mean,

$$
E=\frac{2 E_{\perp}+E_{z}}{3},
$$

and the effective radius of the anisotropic polaron by the geometric mean:

$$
r_{P}=\left(r_{P \perp}^{2} r_{P z}\right)^{1 / 3} .
$$

\section{Polaron formation energy in the generalized Fröhlich cubic model}

The generalized Fröhlich model is considered now in the specific case of cubic materials. The most general case of the Fröhlich model without restriction on the crystallographic systems is discussed elsewhere [26]. In particular the cubic case arrives with certain advantages: the macroscopic dielectric tensor $\epsilon^{\infty}$ and, in the neighborhood of the zone center, the phonon dispersion do not depend on the wave-vector direction. The results are now reformulated to simplify their structure.

Considering both the electron polaron and the hole polaron in cubic materials (corresponding to conduction states and valence states), the variable $\sigma$, that takes value $\sigma=1$ for electron polarons and $\sigma=-1$ for hole polarons, is introduced to characterize the electronic band dispersion, which will be used to ensure the effective mass is always positive at a band extremum.

The gFr Hamiltonian in the cubic case is expressed as follows:

$$
\begin{aligned}
\hat{H}^{\mathrm{gFr}}= & \sum_{\vec{k}, n} \frac{\sigma \vec{k}^{2}}{2 m_{n}^{*}(\hat{k})} \hat{c}_{\vec{k}, n}^{+} \hat{c}_{\vec{k}, n}+\sum_{\vec{q}, j} \omega_{j \mathrm{LO}} \hat{a}_{\vec{q}, j}^{+} \hat{a}_{\vec{q}, j} \\
& +\sum_{\vec{k} n n^{\prime}, \vec{q} j} g^{\mathrm{gFr}}\left(\vec{k} n n^{\prime}, \vec{q} j\right) \hat{c}_{\vec{k}+\vec{q}, n^{\prime}}^{+} \hat{c}_{\vec{k}, n}\left(\hat{a}_{\vec{q}, j}+\hat{a}_{-\vec{q}, j}^{+}\right) .
\end{aligned}
$$

One can point out several aspects in Eq. (43), with respect to the usual Fröhlich Hamiltonian. The electronic effective mass $\hat{k}$ dependence in this case accounts for possible anisotropic behavior of the electronic dispersion. $\hat{k}$ is the normed direction of the $\vec{k}$ vector. Index $n$ accounts for the summation over the electronic bands, thus one can consider possible degenerate states. The summation over $j$ includes the possibility to couple to multiple phonon branches, still considering only the LO type. Most importantly, compared to the basic one-band model, the EPC term summation over the $n$ and $n^{\prime}$ indices now includes interband contribution, if the bands are degenerate at the edge.

The EPC is given by

$$
\begin{aligned}
g^{\mathrm{gFr}}\left(\vec{k} n n^{\prime}, \vec{q} j\right)= & \frac{i}{q} \frac{4 \pi}{\Omega_{0}}\left(\frac{1}{2 \omega_{j \mathrm{LO}} V_{\mathrm{BvK}}}\right)^{1 / 2} \frac{p_{j \mathrm{LO}}}{\epsilon^{\infty}} \\
& \times \sum_{m} s_{n^{\prime} m}\left(\hat{k}^{\prime}\right)\left[s_{n m}(\hat{k})\right]^{*}
\end{aligned}
$$

where $p_{j \mathrm{LO}}$ is the phonon mode polarity and the $s(\hat{k})$ tensors are unitary matrices [26], actually the overlap matrices between a fixed basis of wave functions at $\Gamma$ and the wave functions tending to $\Gamma$ along direction $\hat{k}$. The wave vector $\vec{k}^{\prime}=\vec{k}+\vec{q}$ has direction $\hat{k}^{\prime}$. In the nondegenerate case, $s$ is 1 , irrespective of $\hat{k}$.

The polaron binding energy has been derived within the gFr model [26], taking into account possible wave-vector dependence of the phonon frequencies and mode-polarity vector, as well as anisotropic dielectric tensor. These do not show up in the cubic case, thus Eq. (6) of Ref. [26] reduces to

$$
\begin{aligned}
\mathrm{ZPR}^{\mathrm{gFr}}= & -\sum_{j n} \frac{\sigma}{\sqrt{2} \Omega_{0} n_{\mathrm{deg}}}\left\{\int_{4 \pi} d \hat{k}\left[m_{n}^{*}(\hat{k})\right]^{1 / 2}\right\} \\
& \times\left(\omega_{j \mathrm{LO}}\right)^{-3 / 2}\left(\frac{p_{j \mathrm{LO}}}{\epsilon^{\infty}}\right)^{2}
\end{aligned}
$$

with $n_{\mathrm{deg}}$ the degeneracy of the band extrema and $\omega_{j \mathrm{LO}}$ the $j$ th LO phonon mode.

In view of further developments, and in order to highlight the similarity with Eqs. (7) and (11), we rewrite this expression, and introduce the phonon branch-dependent generalization of the adimensional parameter $\alpha$ :

$$
\alpha_{j}=\left\langle m^{* 1 / 2}\right\rangle\left(2 \omega_{j \mathrm{LO}}\right)^{-1 / 2}\left(\epsilon_{j}^{*}\right)^{-1},
$$

where the average square-root effective mass is

$$
\left\langle m^{* 1 / 2}\right\rangle=\sum_{n} \frac{1}{n_{\mathrm{deg}}}\left\{\frac{1}{4 \pi} \int_{4 \pi} d \hat{k}\left[m_{n}^{*}(\hat{k})\right]^{1 / 2}\right\},
$$

and the phonon branch $j$ contribution to $\epsilon^{*-1}$ is

$$
\epsilon_{j}^{*-1}=\frac{4 \pi}{\Omega_{0}}\left(\frac{p_{j \mathrm{LO}}}{\omega_{j \mathrm{LO}} \epsilon^{\infty}}\right)^{2} .
$$

One can check that the static dielectric constant is the sum of the electronic (optical) one and those of all phonon branches $[26,46]$ :

$$
\left(\epsilon^{0}\right)^{-1}=\left(\epsilon^{\infty}\right)^{-1}+\sum_{j}\left(\epsilon_{j}^{*}\right)^{-1} .
$$

Such formulas generalize easily to the noncubic case, but the cubic case allows a decoupling of the electronic directionality average from the vibrational and dielectric behavior.

In terms of these quantities, the polaron binding energy in the generalized cubic Fröhlich model is expressed as

$$
\begin{aligned}
\mathrm{ZPR} & =-\sigma \sum_{j} \alpha_{j} \omega_{j \mathrm{LO}} \\
& =-\sigma\left\langle m^{* 1 / 2}\right\rangle \sum_{j}\left(2 \omega_{j \mathrm{LO}}\right)^{-1 / 2}\left(\epsilon_{j}^{*}\right)^{-1} \omega_{j \mathrm{LO}} .
\end{aligned}
$$

In what follows, the vibrational and dielectric factor in this expression, as well as a similar expression without the rightmost factor $\omega_{j \mathrm{LO}}$, will be encountered often, so that we define, for $n=0$ and 1 ,

$$
V_{n}=\sum_{j}\left(2 \omega_{j \mathrm{LO}}\right)^{-1 / 2}\left(\epsilon_{j}^{*}\right)^{-1}\left(\omega_{j \mathrm{LO}}\right)^{n},
$$

which gives

$$
\mathrm{ZPR}=-\sigma\left\langle m^{* 1 / 2}\right\rangle V_{1}
$$




\section{POLARON EFFECTIVE MASS IN CUBIC MATERIALS}

We now derive the expressions for the polaron effective mass, in the weak-coupling limit, from many-body perturbation theory. The self-energy is obtained, then derived twice with respect to the wave vector, giving the effective mass. We will treat first the nondegenerate case, then the degenerate case.

\section{A. Effective masses in the nondegenerate case}

In order to determine the polaron effective mass, the selfenergy $\Sigma(\vec{k})$ is determined at the lowest order in many-body perturbation theory, in the on-the-mass-shell approach. The polaron energy $E_{P}(\vec{k})$ is obtained as

$$
E_{P}(\vec{k})=E(\vec{k})+\Sigma(\vec{k}, E(\vec{k})),
$$

with

$$
\Sigma(\vec{k}, z)=\frac{1}{\pi} \sum_{j} \int d^{3} q \frac{\omega_{j \mathrm{LO}}}{4 \pi q^{2} \epsilon_{j}^{*}} \frac{1}{[z-E(\vec{k}+\vec{q})]-\sigma \omega_{j \mathrm{LO}}},
$$

where $E(\vec{k})$ is the bare electron or hole energy depending on the wave vector $\vec{k}$.

In the nondegenerate case, the bare electronic energy is expressed in terms of effective masses along the three principal axes of the electronic dispersion relation (with the general case of a band edge anywhere in the Brillouin zone, note that cross terms like $m_{x y}$ can always be eliminated by the proper choice of axes). Deviations from quadratic behavior are ignored, and are beyond the gFr model. Thus,

$$
E(\vec{k})=\frac{\sigma}{2}\left(\frac{k_{x}^{2}}{m_{x}^{*}}+\frac{k_{y}^{2}}{m_{y}^{*}}+\frac{k_{z}^{2}}{m_{z}^{*}}\right) .
$$

After expanding the self-energy in a Taylor series up to second order in the wave-vector components, the polaron dispersion energy in the nondegenerate energy case becomes

$$
E_{P}(\vec{k}) \approx \Sigma(0, \vec{k}=0)+\frac{\sigma}{2}\left(\frac{k_{x}^{2}}{m_{P, x}^{*}}+\frac{k_{y}^{2}}{m_{P, y}^{*}}+\frac{k_{z}^{2}}{m_{P, z}^{*}}\right),
$$

where $\Sigma(0, \vec{k}=0)$ is the ZPR energy. The quantities $m_{P, i}^{*}$ are the sought polaron effective masses:

$$
\frac{1}{m_{P, i}^{*}}=\frac{1}{m_{i}^{*}}+\left.\sigma \frac{d^{2} \Sigma(\vec{k}, E(\vec{k}))}{d k_{i}^{2}}\right|_{k=0} ; \quad i=x, y, z .
$$

Equations (53)-(57) can be worked out as follows. One defines

$$
I_{P}\left(\vec{k}, \omega_{j \mathrm{LO}}, m_{i}^{*}\right)=\int \frac{d^{3} q}{4 \pi q^{2}} \frac{1}{\sigma[E(\vec{k})-E(\vec{k}+\vec{q})]-\omega_{j \mathrm{LO}}},
$$

with the dependence of $I_{P}$ on effective masses being given by Eq. (55), so that

$$
\Sigma(\vec{k}, E(\vec{k}))=\frac{\sigma}{\pi} \sum_{j} \frac{\omega_{j \mathrm{LO}}}{\epsilon_{j}^{*}} I_{P}\left(\vec{k}, \omega_{j \mathrm{LO}}, m_{i}^{*}\right) .
$$

One easily proves that

$$
I_{P}\left(0, \omega_{j \mathrm{LO}}, m_{i}^{*}\right)=\left(\omega_{j \mathrm{LO}}\right)^{-1 / 2} I_{P}\left(0,1, m_{i}^{*}\right)
$$

and

$$
\left.\frac{\partial^{2} I_{P}}{\partial k_{i}^{2}}\right|_{k=0}=-\left.2 \frac{\partial^{2} I_{P}}{\partial \omega_{j \mathrm{LO}} \partial m_{i}^{*}}\right|_{k=0, \omega_{j \mathrm{LO}}}
$$

giving

$$
\left.\frac{d^{2} \Sigma(\vec{k}, E(\vec{k}))}{d k_{i}^{2}}\right|_{k=0}=\left.\frac{\sigma}{\pi} \frac{\partial I_{P}}{\partial m_{i}^{*}}\right|_{k=0, \omega=1} V_{0},
$$

to be inserted in Eq. (57). In this expression, the decomposition between dependence on the electronic dispersion, through the effective masses, and the dependence on the phonon branches is clear.

The required expression for $I_{P}\left(0,1, m_{i}^{*}\right)$, namely,

$$
I_{P}\left(0,1, m_{i}^{*}\right)=-\int \frac{d^{3} q}{4 \pi q^{2}} \frac{1}{\frac{1}{2}\left(\frac{q_{x}^{2}}{m_{x}^{*}}+\frac{q_{y}^{2}}{m_{y}^{*}}+\frac{q_{z}^{2}}{m_{z}^{*}}\right)+1},
$$

relates directly to the average square-root effective mass, Eq. (47), in this nondegenerate case. Indeed, $\vec{q}$ can be decomposed in its norm $q$ and direction $\hat{q}, \vec{q}=q \hat{q}$, the radial and angular integrations can be decoupled, and the radial one can be performed exactly. The effective mass in direction $\hat{q}$ is given by

$$
\frac{1}{m^{*}(\hat{q})}=\frac{\hat{q}_{x}^{2}}{m_{x}^{*}}+\frac{\hat{q}_{y}^{2}}{m_{y}^{*}}+\frac{\hat{q}_{z}^{2}}{m_{z}^{*}},
$$

and one obtains

$$
I_{P}\left(0,1, m_{i}^{*}\right)=-\frac{\pi}{\sqrt{2}}\left\langle m^{* 1 / 2}\right\rangle
$$

Finally, one recovers first Eq. (50), as ZPR $=\Sigma(\overrightarrow{0}, E(\overrightarrow{0}))$, but one finds also

$$
\frac{1}{m_{P, i}^{*}}=\frac{1}{m_{i}^{*}}-\frac{\partial\left\langle m^{* 1 / 2}\right\rangle}{\partial m_{i}^{*}} V_{0}
$$

In the nondegenerate isotropic case, e.g., when one has a single electronic band edge at $\Gamma$, the polaron effective mass simplifies to Eq. (12).

Moreover, one can treat also analytically the nondegenerate anisotropic uniaxial case, suitable to describe materials where the band extremum is found at $X$ or $L$ points in cubic materials, with a uniaxial electronic dispersion relation. In this case, we define

$$
m_{x}=m_{y}=m_{\perp}
$$

as well as the ratio between effective masses $\mu^{*}=\frac{m_{\perp}}{m_{z}}$. We can distinguish two possible scenarios, $\mu^{*}>1$ and $\mu^{*}<1$. The self-energy will inherit the uniaxial symmetry of the electronic structure and we can determine the in-plane and out-of-plane derivatives based solely on the two different electronic effective masses. Mathematically, one obtains the following formulas. The average square root of the effective mass over all directions becomes

$$
\left\langle m^{* 1 / 2}\right\rangle=m_{\perp}^{1 / 2} S\left(\mu^{*}-1\right)
$$

where

$$
S(x)= \begin{cases}\frac{\operatorname{arcsinh}\left(x^{1 / 2}\right)}{x^{1 / 2}}, & x>0, \\ \frac{\arcsin \left[(-x)^{1 / 2}\right]}{(-x)^{1 / 2}}, & x<0 .\end{cases}
$$




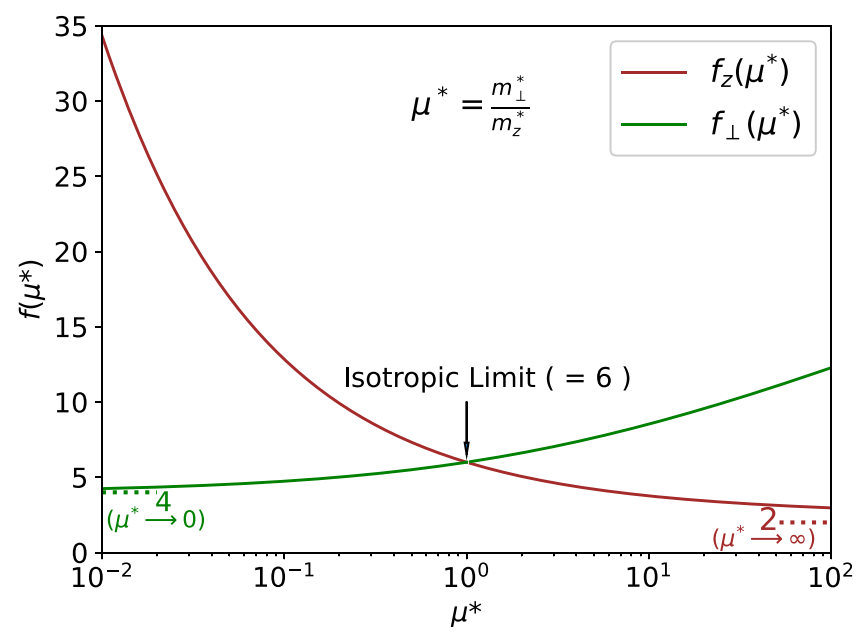

FIG. 1. Polaron effective mass breakdown limit in the uniaxial case. Note that when the two relevant effective masses $m_{z}^{*}$ and $m_{\perp}^{*}$, respectively, are equal we reach the isotropic limit breakdown of 6 .

This yields analytical formulas for the electron-phonon modification of the inverse effective masses:

$$
\left.\frac{d^{2}}{d k_{\perp}^{2}} \Sigma\right|_{k=0}=\sigma \frac{1}{4 \sqrt{2}} m_{\perp}^{-1 / 2}\left[\frac{S\left(\mu^{*}-1\right)-\left(\mu^{*}\right)^{1 / 2}}{\mu^{*}-1}\right] V_{0}
$$

and

$$
\left.\frac{d^{2}}{d k_{z}^{2}} \Sigma\right|_{k=0}=\sigma \frac{1}{2 \sqrt{2}} m_{z}^{-1 / 2} \frac{\mu^{*}}{\mu^{*}-1}\left[1-\mu^{* 1 / 2} S\left(\mu^{*}-1\right)\right] V_{0} .
$$

The polaron effective mass is shown to reach the perturbation theory breakdown limit at a different rate when compared to the isotropic case, as it depends on the direction in the uniaxial case. The self-energy behaves differently along $\perp$ and $z$ directions, as shown in Eqs. (70) and (71), and the polaron effective mass has the same behavior:

$$
m_{P, \perp}^{*}=m_{\perp}^{*}\left(1-\frac{1}{f_{\perp}\left(\mu^{*}\right)} \Sigma_{i} \alpha_{i}\right)^{-1}
$$

and

$$
m_{P, z}^{*}=m_{z}^{*}\left(1-\frac{1}{f_{z}\left(\mu^{*}\right)} \Sigma_{i} \alpha_{i}\right)^{-1}
$$

with

$$
f_{\perp}\left(\mu^{*}\right)=4\left(1-\mu^{*}\right)\left[1-\frac{\left(\mu^{*}\right)^{1 / 2}}{S\left(\mu^{*}-1\right)}\right]^{-1}
$$

and

$$
f_{z}\left(\mu^{*}\right)=2 \frac{\left(1-\mu^{*}\right)}{\mu^{*}}\left[\frac{\left(\mu^{*}\right)^{-1 / 2}}{S\left(\mu^{*}-1\right)}-1\right]^{-1} .
$$

Written as above, one sees that the effective mass changes sign when the relevant $f\left(\mu^{*}\right)$ function equals $\Sigma_{i} \alpha_{i}$. In the isotropic case, $\mu^{*}=1, f(1)=6$, which recovers the $\alpha=6$ limit for the perturbation theory breakdown. Figure 1 shows that the mass breakdown limit is reached earlier when moving away from the isotropic case $\left(\mu^{*}=1\right)$. In the low physical limit of $\mu^{*}$, the breakdown is dominated by the mass enhancement to the in-plane electronic effective mass, $m_{\perp}^{*}$, reaching a limit as low as 4, while in the high physical limit the limitation comes from the out-of-plane mass enhancement, with an even lower limit of 2. We recall that, as in the standard Fröhlich model, the breakdown of perturbation theory does not necessarily entail a true divergence or pathology of the physical system.

\section{B. Effective masses in the threefold degenerate case}

The top valence bands of many cubic materials present a threefold degeneracy. The degeneracy will be lifted when the wave vector departs from the band extremum (splitting either $2+1$ or $1+1+1$ depending on the direction). This behavior follows the general description of band structures around degenerate extrema, given by Luttinger and Kohn [47]. Taking the extremum eigenvalue as a reference, the bare electronic energy dispersion is described by

$$
E_{n}(\vec{k})=\sigma \frac{k^{2}}{2 m_{n}^{*}(\vec{k})},
$$

up to quadratic order, with a direction- and band-dependent effective mass $m_{n}^{*}(\hat{k})$. Such an effective mass fulfills the following eigenvalue equation:

$$
H_{\mathrm{LK}}(\vec{k}) \vec{e}_{n}(\hat{k})=\frac{k^{2}}{2 m_{n}^{*}(\hat{k})} \vec{e}_{n}(\hat{k}),
$$

where $H_{\mathrm{LK}}$ is the Luttinger-Kohn (LK) Hamiltonian matrix the dimension of which is equal to the degeneracy of the problem, $\vec{k}=k \cdot \hat{k}$, and $n$ is the band index. This matrix is written referring to a fixed basis set for the wave functions which forms an irreducible representation of the symmetry group of the extremum wave vector. The actual $\hat{k}$-dependent eigenfunction for band $n$ in direction $\hat{k}$ is obtained from the eigenvector $\vec{e}_{n}(\hat{k})$.

Specifically, in the cubic three-band degenerate case, the Luttinger-Kohn Hamiltonian is written [47]

$$
\begin{aligned}
& H_{\mathrm{LK}}(\vec{k}) \\
& \quad=\left(\begin{array}{ccc}
A k_{x}^{2}+B\left(k_{y}^{2}+k_{z}^{2}\right) & C k_{x} k_{y} & C k_{x} k_{z} \\
C k_{x} k_{y} & A k_{y}^{2}+B\left(k_{z}^{2}+k_{x}^{2}\right) & C k_{y} k_{z} \\
C k_{x} k_{z} & C k_{y} k_{z} & A k_{z}^{2}+B\left(k_{x}^{2}+k_{y}^{2}\right)
\end{array}\right),
\end{aligned}
$$

with three parameters $A, B$, and $C$.

Turning on the electron-phonon coupling, the polaron dispersion relation in the degenerate band case will have the same behavior, including the same symmetry characteristics, which gives, similarly to Eqs. (53) and (56),

$$
\begin{aligned}
E_{P, n}(\vec{k}) & =\sigma \frac{k^{2}}{2 m_{n}^{*}(\vec{k})}+\Sigma_{n}(\vec{k}) \\
& \approx \Sigma_{n}(\vec{k}=0)+\sigma \frac{k^{2}}{2 m_{P, n}^{*}(\hat{k})}
\end{aligned}
$$


with

$$
\frac{1}{m_{P, n}^{*}(\hat{k})}=\frac{1}{m_{n}^{*}(\hat{k})}+\left.\sigma \frac{d^{2} \Sigma_{n}\left(\vec{k}, E_{n}(\vec{k})\right)}{d k^{2}}\right|_{k=0, \vec{k}=k . \hat{k}} .
$$

Due to the electron-phonon interaction, the bare effective electronic mass $m_{n}^{*}(\vec{k})$ is replaced by the polaron effective mass $m_{P, n}^{*}(\vec{k})$.

The behavior of the effective masses with respect to the direction $\vec{k}$ is far from trivial, and generates a so-called warping of the band structure, which has been analyzed in detail by Mecholsky et al. [28]. For further reference, we list the inverse of effective masses and degeneracies along the (100), (110), and (111) high-symmetry directions:

$$
\begin{aligned}
m_{n}^{*-1}(100)= & 2 A \text { or } 2 B(\text { twofold }), \\
m_{n}^{*-1}(110)= & (A+B+C),(A+B-C) \text { or } 2 B, \\
m_{n}^{*-1}(111)= & \frac{2}{3}(A+2 B+2 C) \text { or } \\
& \frac{2}{3}(A+2 B-C)(\text { twofold }) .
\end{aligned}
$$

The maximal and minimal effective masses are found along these three directions, depending on the ratio between Luttinger-Kohn parameters. The self-energy, in the on-themass-shell approach, is similar to that for the nondegenerate case, although one has to generalize the denominator of Eq. (54) from scalar quantities to matrix quantities, as follows:

$$
\begin{aligned}
\Sigma_{n}(\vec{k}, & \left.E_{n}(\vec{k})\right) \\
= & \frac{1}{\pi} \sum_{j} \int d^{3} q \frac{\omega_{j \mathrm{LO}}}{4 \pi q^{2} \epsilon_{j}^{*}} \vec{e}_{n}^{T}(\hat{k}) \\
& \times\left(\frac{k^{2}}{2 m_{n}(\vec{k})} \mathbb{I}-H(\vec{k}+\vec{q})-\sigma \omega_{j \mathrm{LO}} \mathbb{I}\right)^{-1} \vec{e}_{n}(\hat{k}) .
\end{aligned}
$$

In order to characterize the polaron effective masses, instead of working with a particular band, it is easier to work with the direct generalization of the LK Hamiltonian (which is obtained from generic symmetry considerations) to the electron-phonon case, with modified values of the $A, B$, and $C$ parameters, namely, $A_{P}, B_{P}$, and $C_{P}$.

As an important property of the LK Hamiltonian, the sum of the effective mass inverses for the three bands gives the trace of the LK matrix, which amounts to a constant value, namely, $(A+2 B) k^{2}$, independently of the wave-vector direction. This is indeed fulfilled in Eq. (81). As a consequence the sum of the polaron effective mass inverses also amounts to a constant value, $2\left(A_{P}+2 B_{P}\right)$, irrespective of the direction of the polaron energy dispersion.

We now examine specific cases. When the Luttinger-Kohn parameter $C$ vanishes, the electronic dispersion relation corresponds to the absence of coupling between the top (bottom) three valence (conduction) bands: the $H_{\mathrm{LK}}$ matrix is diagonal. The eigenvectors of Eq. (77) are unit vectors for bands 1, 2, and 3 , irrespective of the direction $\hat{k}$. The electronic effective masses for the three disjoint bands are determined by the values of the remaining Luttinger parameters, with $m_{\perp}^{-1}=2 B$ and $m_{z}^{-1}=2 A$, interestingly falling back on the nondegenerate uniaxial case, for each band. Thus the analysis of Sec. III A applies.
In contrast, if $A=B+C$, one obtains the isotropic threefold degenerate case, in which, irrespective of the wave-vector direction, there is a nondegenerate band, with inverse effective mass $m_{0}^{-1}=2 A$ and two degenerate bands with effective mass $m_{1}^{-1}=2 B$. This case has been tackled by Trebin and Rössler [32], who provide the analytical expression for the self-energy and effective masses in the one-phonon branch hypothesis. The present formalism delivers exactly the same analytical expressions for the effective masses in such case, but also generalizes them to the multiphonon case. Explicitly,

$$
\mathrm{ZPR}=-\frac{\sigma}{3}\left[\left(m_{0}^{*}\right)^{1 / 2}+2\left(m_{1}^{*}\right)^{1 / 2}\right] V_{1},
$$

in line with Eq. (52), and for the polaron effective masses

$$
\frac{1}{m_{P, 0}^{*}}=\frac{1}{m_{0}^{*}}+\left[\frac{3}{10}\left(m_{0}^{*}\right)^{-1 / 2}-\frac{2}{15}\left(m_{1}^{*}\right)^{-1 / 2}-\frac{\left(m_{1}^{*}\right)^{1 / 2}}{3 m_{0}^{*}}\right] V_{0}
$$

and

$$
\begin{aligned}
\frac{1}{m_{P, 1}^{*}}=\frac{1}{m_{1}^{*}}+ & {\left[-\frac{1}{15}\left(m_{0}^{*}\right)^{-1 / 2}\right.} \\
& \left.+\frac{1}{15}\left(m_{1}^{*}\right)^{-1 / 2}-\frac{\left(m_{0}^{*}\right)^{1 / 2}}{6 m_{1}^{*}}\right] V_{0},
\end{aligned}
$$

In all cases except $C=0$ and $A=B+C$, as soon as $n>1$, Eq. (82) must be evaluated numerically, first treating the computation of the self-energy as a function of the wave vector, and then taking its second-order derivative, as needed in Eq. (80).

In order to calculate the self-energy in the degenerate band case, we perform a numerical integration over $\vec{q}$ expressed in spherical coordinates:

$$
\begin{aligned}
\Sigma_{n}\left(\vec{k}, E_{n}(\vec{k})\right) \\
=\frac{1}{\pi} \sum_{j} \frac{\omega_{j \mathrm{LO}}}{\epsilon_{j}^{*}} \int_{0}^{\infty} d q \frac{1}{4 \pi q^{2}} \int_{4 \pi} d \hat{q} \vec{e}_{n}^{T}(\hat{k}) \\
\quad \times\left(\frac{k^{2}}{2 m_{n}(\vec{k})} \mathbb{I}-H(\vec{k}+\vec{q})-\sigma \omega_{j \mathrm{LO}} \mathbb{I}\right)^{-1} \vec{e}_{n}(\hat{k})
\end{aligned}
$$

with

$$
\vec{q}=q \hat{q}=q\left(\begin{array}{c}
\sin \theta \cos \phi \\
\sin \theta \sin \phi \\
\cos \theta
\end{array}\right)
$$

Regarding the integral over $q \in[0, \infty)$, one could use a homogeneous grid integration method, with the maximal value $q_{\max }$ tending to infinity. However, such homogeneous grid integration approach converges slowly. Instead, the semi-infinite domain $q \in[0, \infty)$ can be mapped onto a finite one given that the behavior of the integrand follows $f(q) \rightarrow \frac{1}{\alpha^{2}+\beta^{2} q^{2}}$, for large $q$. One performs the following change of variable:

$$
q=\left(\frac{\omega_{\mathrm{LO}}}{\gamma}\right)^{1 / 2} \tan \xi
$$

giving

$$
\frac{d q}{d \xi}=\left(\frac{\omega_{\mathrm{LO}}}{\gamma}\right)^{1 / 2} \frac{1}{\cos ^{2} \xi}=\left(\frac{\omega_{\mathrm{LO}}}{\gamma}\right)^{1 / 2}\left(\frac{\gamma q^{2}}{\omega_{\mathrm{LO}}}+1\right)
$$


and

$$
\int_{0}^{\infty} d q f(q)=\int_{0}^{\pi / 2} d \xi \frac{d q}{d \xi} f[q(\xi)],
$$

which can easily be integrated numerically, as the additional factor in the integrand, $\frac{d q}{d \xi}$, counterbalances the behavior of $f(q)$, giving a smooth integrand in the interval $[0, \pi / 2] . \gamma$ can be chosen arbitrarily, but the integral converges quickly if it is chosen as the $A$ Luttinger parameter.

For the $\phi$ integration, we use $N_{\phi}$ points, regularly spaced, with appropriate weights, while the integral over $\theta$ is performed in terms of a uniform grid of $\cos (\theta)$ using $N_{\theta}$ points, also with appropriate weights (see Supplemental Material for details [48]). One might instead use the Gauss-Legendre technique for the $\cos (\theta)$ integral. Although it has not been used in the present paper, the implementation available in the latest version of ABINIT relies on this more efficient approach.

\section{POLARON LOCALIZATION LENGTHS IN CUBIC MATERIALS}

Similarly to the case of effective masses, we will examine the nondegenerate situation first, then the degenerate case, with particular focus on the threefold degeneracy.

\section{A. Polaron localization lengths in the nondegenerate case}

In Sec. II A the polaron radius for the isotropic electronic dispersion was considered, for the case with only one phonon branch. In the present section, the electronic dispersion is anisotropic, with three different effective masses along the three principal axes, and several phonon branches are included.

The variational energy is again a sum of electronic and epp contributions, as written in Eq. (13). Due to the anisotropy of the effective masses, $E_{\mathrm{el}}$ becomes

$$
E_{\mathrm{el}}[\phi(\vec{r})]=\int d \vec{r} \sum_{i} \frac{1}{2 m_{i}}\left|\frac{\partial \phi(\vec{r})}{\partial r_{i}}\right|^{2}
$$

for $i=x, y, z$. The density construction, Eqs. (15) and (16), is unchanged. The epp energy Eq. (17) now includes contributions from several phonon modes, labeled $j$ :

$$
E_{e p p}[\phi(\vec{r})]=-\sum_{j} \int d \vec{k} \frac{4 \pi}{2 k^{2}}\left(\epsilon_{j}^{*}\right)^{-1} \rho^{*}(\vec{k}) \rho(\vec{k}) .
$$

The multiphonon situation is actually quite easy to treat in this strong-coupling approach, as one can define

$$
\left(\epsilon^{*}\right)^{-1}=\sum_{j}\left(\epsilon_{j}^{*}\right)^{-1}
$$

and recover Eq. (17).

Following the same line of thought as in Sec. II A, a (anisotropic) Gaussian variational wave function ansatz can be considered as follows:

$$
\phi(x, y, z)=\left(\frac{1}{\pi^{3 / 2} a_{P}^{3}}\right)^{1 / 2} e^{-\frac{1}{2}\left(\frac{x^{2}}{a_{P x}^{2}}+\frac{y^{2}}{a_{P y}^{2}}+\frac{z^{2}}{a_{P z}^{2}}\right)},
$$

where $a_{P x}, a_{P y}$, and $a_{P z}$ are characteristic localization lengths along the $x, y$, and $z$ directions, and $a_{P}$ is the volumetric mean radius of the polaron, that is, the radius of the sphere that gives the same volume as that of the ellipsoid built on the semiaxes $a_{P x}, a_{P x}$, and $a_{P x}$ :

$$
a_{P}^{3}=a_{P x} a_{P y} a_{P z} .
$$

With such a trial wave function, $E_{\mathrm{el}}$ and $E_{\text {epp }}$ become

$$
E_{\mathrm{el}}=\frac{1}{4}\left(\frac{1}{a_{P x}^{2} m_{x}^{*}}+\frac{1}{a_{P y}^{2} m_{y}^{*}}+\frac{1}{a_{P z}^{2} m_{z}^{*}}\right)
$$

and

$$
\begin{aligned}
E_{\text {epp }}= & -\frac{1}{\epsilon^{*}(2 \pi)^{1 / 2}} \\
& \times \frac{1}{4 \pi} \int_{4 \pi} d \hat{k}\left(a_{P x}^{2} \hat{k}_{x}^{2}+a_{P y}^{2} \hat{k}_{y}^{2}+a_{P z}^{2} \hat{k}_{z}^{2}\right)^{-1 / 2} .
\end{aligned}
$$

A polaron directional localization length $a_{P d}(\hat{k})$ is then defined as

$$
a_{P d}(\hat{k})=\left(a_{P x}^{2} \hat{k}_{x}^{2}+a_{P y}^{2} \hat{k}_{y}^{2}+a_{P z}^{2} \hat{k}_{z}^{2}\right)^{1 / 2},
$$

the angular average of its inverse being

$$
\left\langle a_{P d}^{-1}\right\rangle_{4 \pi}=\frac{1}{4 \pi} \int_{4 \pi} d^{3} k\left[a_{P d}(\hat{k})\right]^{-1},
$$

so that $E_{\text {epp }}$ can be written in terms of this average:

$$
E_{e p p}=-\frac{\left\langle a_{P d}^{-1}\right\rangle_{4 \pi}}{\epsilon^{*}(2 \pi)^{1 / 2}} .
$$

Minimization of $E_{\mathrm{el}}+E_{\text {epp }}$ by variation of the localization lengths $a_{P i}$, for given $\epsilon^{*}$ and $m_{i}^{*}$, delivers the optimal Gaussian trial wave function.

This optimization problem does not have an analytical solution in the most general case, but can be treated numerically, either by direct minimization of $E$ in the space of $a_{P i}$, or by requiring that the derivatives of $E$ with respect to $a_{P i}$ vanish, which amounts to

$$
\frac{1}{m_{i}^{*}}=-\frac{2 a_{P i}^{3}}{\epsilon^{*}(2 \pi)^{1 / 2}} \frac{\partial\left\langle a_{P d}^{-1}\right\rangle_{4 \pi}}{\partial a_{P i}} .
$$

As for the effective masses, a specific treatment of the uniaxial case is possible. It does not deliver explicit values for the localization lengths as a function of $\epsilon$ and $m_{i}^{*}$, but an implicit relationship, as follows. The convention for the $x$ and $y$ masses is given by Eq. (67). By symmetry, the $x$ and $y$ localization lengths must be equal, with

$$
a_{P x}=a_{P y}=a_{P \perp},
$$

We define the anisotropy parameter $\delta$,

$$
\delta=\frac{a_{P z}^{2}}{a_{P \perp}^{2}}-1,
$$

and arrive at relatively simple and intuitive formulas for the inplane, $a_{P \perp}$, and out-of-plane, $a_{P z}$, polaron localization lengths as functions of $a_{P}$ and $\delta$ :

$$
\begin{gathered}
a_{P \perp}=a_{P}(1+\delta)^{-1 / 6}, \\
a_{P z}=a_{P}(1+\delta)^{1 / 3} .
\end{gathered}
$$


After some algebra, the energy to be minimized as a function of $a_{P}$ and $\delta$ is written

$$
\begin{aligned}
E_{P}= & \frac{1}{4 a_{P}^{2}}\left(\frac{2(1+\delta)^{1 / 3}}{m_{\perp}^{*}}+\frac{(1+\delta)^{-2 / 3}}{m_{z}^{*}}\right) \\
& -\frac{1}{\epsilon^{*}(2 \pi)^{1 / 2} a_{P}}(1+\delta)^{1 / 6} S(\delta),
\end{aligned}
$$

where the $S$ function is defined in Eq. (69).

Optimization with respect to $a_{P}$ is easy, while the determination of $\delta$ is more delicate. Considering $\delta$ as the basic variable, the effective mass ratio $\mu^{*}=\frac{m_{\perp}}{m_{z}}$ can be determined analytically:

$$
\mu^{*}(\delta)=\left(S\left(\frac{d S}{d \delta}\right)+\frac{1}{2(1+\delta)}\right)^{-1}-2(1+\delta),
$$

where the derivative of the $S$ function is

$$
\frac{d S}{d \delta}=\frac{1}{2 \delta(1+\delta)^{1 / 2}}-\frac{S(\delta)}{2 \delta} .
$$

An explicit solution of the optimization problem would require $\delta$ as a function of $\mu^{*}$, but unfortunately Eq. (107) cannot be inverted analytically. Still, the polaron localization length and energy can be determined as a function of $\delta$ as well, so that there is a parametric relationship between the polaron characteristics and $\mu^{*}$.

Compared to the spherical (isotropic) scenario, Eq. (19), the spherical average of the polaron localization length is now

$$
a_{P}=\frac{3}{2} \sqrt{2 \pi}\left\langle m^{* 1 / 2}\right\rangle^{-2} \epsilon^{*} D(\delta)
$$

where $D(\delta)$ is the enhancement factor with respect to the isotropic case. Its expression is

$$
D(\delta)=\frac{1}{3}(1+\delta)^{-5 / 6}\left(\frac{d S}{d \delta}+\frac{S(\delta)}{2(1+\delta)}\right)^{-1} .
$$

In Fig. 2 we show the in-plane, $a_{p_{\perp}}$, and out-of-plane, $a_{p_{z}}$, localization lengths expressed in terms of $\mu^{*}$. For $\mu^{*}$ smaller than 1 , the smallest polaron localization length is $a_{p_{z}}$, while for $\mu^{*}$ larger than $1 a_{p_{\perp}}$ is the smallest. For $\mu^{*}=10^{4}$, the value of $a_{p_{\perp}}$ is roughly one order of magnitude smaller than $a_{p_{z}}$.

Finally the polaron formation energy in the uniaxial case can be expressed in terms of the adimensional parameters $\delta$ and $\mu^{*}(\delta)$ and one of the dimensional parameters $m_{\perp}^{*},\left\langle m^{* 1 / 2}\right\rangle$, or even $a_{P}$ :

$$
E(\delta)=-\frac{1}{4 \pi} \epsilon^{*-2} m_{\perp}^{*}[S(\delta)]^{2}\left(1+\frac{\mu^{*}}{2(1+\delta)}\right)^{-1},
$$

or, equivalently,

$$
E(\delta)=-\frac{1}{4 \pi}\left(\frac{\left\langle m^{* 1 / 2}\right\rangle S(\delta)}{\epsilon^{*} S\left(\mu^{*}-1\right)}\right)^{2}\left(1+\frac{\mu^{*}}{2(1+\delta)}\right)^{-1}
$$

or

$$
E(\delta)=-\frac{3 \sqrt{2 \pi}}{8 \pi} \frac{D(\delta)}{\epsilon^{*} a_{P}}\left(\frac{S(\delta)}{S\left(\mu^{*}-1\right)}\right)^{2}\left(1+\frac{\mu^{*}}{2(1+\delta)}\right)^{-1} .
$$

Numerically, it is then easy to represent $E$ as a function of $\mu^{*}$ only, either fixing $m_{\perp}^{*},\left\langle m^{* 1 / 2}\right\rangle$, or $a_{P}$. The polaron formation energy in the latter case is represented in Fig. 3.

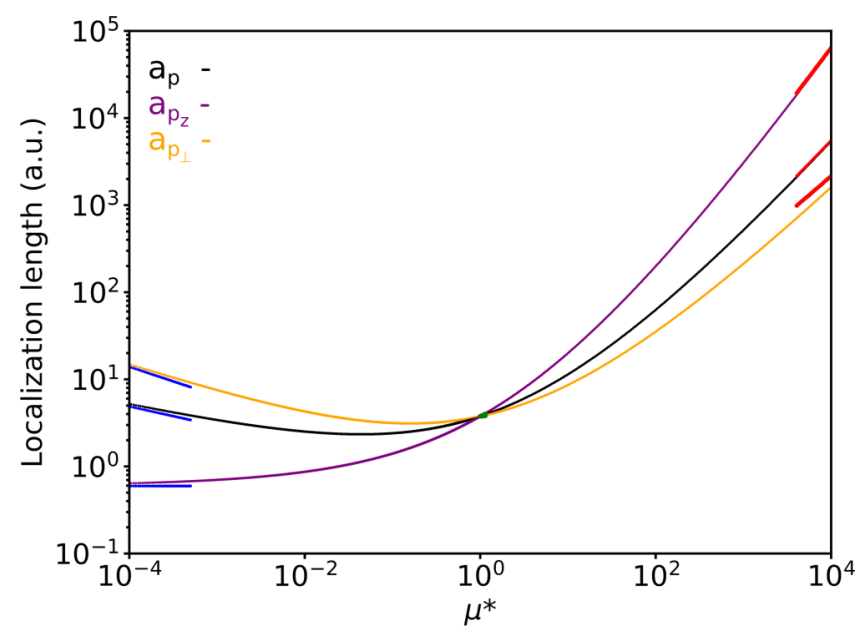

FIG. 2. Polaron localization length in the uniaxial limit calculated at constant density of states expressed in terms of $\mu^{*}$ for the volumetric average case $\left(a_{P}\right)$, the out-of-plane localization length $\left(a_{P z}\right)$, and the in-plane localization length $\left(a_{P \perp}\right)$. In the low limit of $\mu^{*}$, the asymptotic analytical behavior is represented in blue. In the $\mu^{*} \rightarrow 1$ limit, the analytical expression is represented in green. Finally, in the large $\mu^{*}$ limit, the asymptotic behavior analytical limit is represented in red. The expressions for the analytical asymptotic behavior in the three cases are provided in the Supplemental Material [48]. We consider here $\epsilon^{*}=1$ and $m_{\text {DoS }}^{*}=1$ (see definition in Supplemental Material).

\section{B. Polaron localization lengths in the degenerate case}

In the degenerate case, the polaronic wave function includes several components, namely, a superposition of contributions from the different degenerate electronic bands. We introduce the band label $j$ that runs from 1 to $n$, and note the

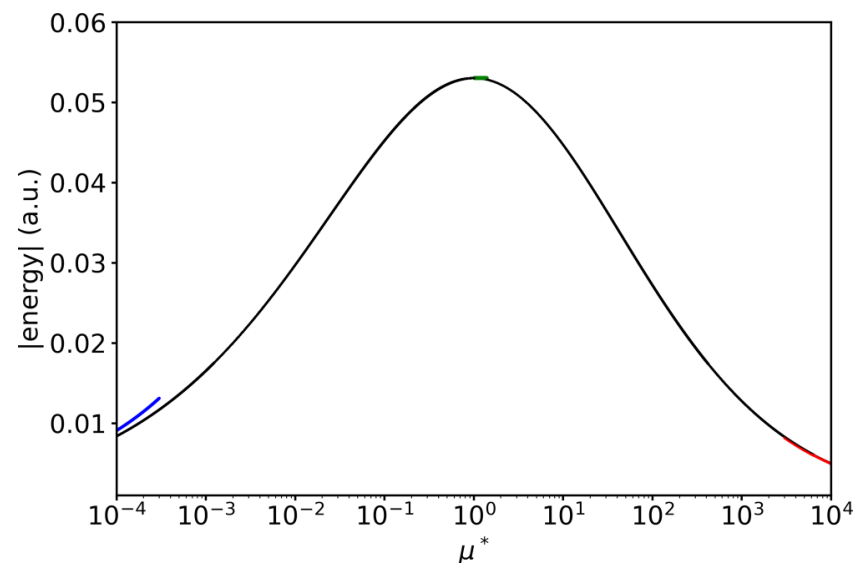

FIG. 3. Absolute value of the polaron formation energy in the uniaxial limit calculated at constant volumetric localization length (or spherical average $a_{P}$ ) as a function of $\mu^{*}$. In the low limit of $\mu^{*}$, the asymptotic analytical behavior is represented in blue. In the spherical limit, the analytical expression is represented in green. Finally, in the large $\mu^{*}$ limit, the asymptotic behavior analytical limit is represented in red. The expressions for the analytical asymptotic behavior in the three cases are provided in the Supplemental Material [48]. We consider $\epsilon^{*}=1$ and $a_{P}=1$ (see definition in Supplemental Material). 
components of the wave function $\phi_{j}(\vec{r})$. The electronic density is formed by summing the densities from all components,

$$
\rho(\vec{r})=\sum_{j}\left|\phi_{j}(\vec{r})\right|^{2}
$$

and contributes to the $E_{e p p}$ in the same way as in the nondegenerate case, even if there are several phonon branches. The electronic energy, $E_{\mathrm{el}}$, is also obtained from the contribution of the different components, through the Luttinger-Kohn Hamiltonian. Expressed in reciprocal space,

$$
E_{\mathrm{el}}\left[\phi_{j}\right]=\left.\sigma \int d^{3} k \sum_{j, j^{\prime}=1}^{n} \phi_{j}^{*}(\vec{k}) H_{\mathrm{LK}}(\vec{k})\right|_{\left(j, j^{\prime}\right)} \phi_{j^{\prime}}(\vec{k}) .
$$

Moreover, the multicomponent wave function must be normalized:

$$
1=\sum_{j=1}^{n} \int d^{3} k \phi_{j}^{*}(\vec{k}) \phi_{j}(\vec{k})=\sum_{j=1}^{n} \int d^{3} r \phi_{j}^{*}(\vec{r}) \phi_{j}(\vec{r}) .
$$

Like in the nondegenerate case, we are not looking for an exact solution of this minimization problem, but we are trying to find the best trial wave function among a set of acceptable wave functions with some adjustable parameters.

In particular, since the $E_{e p p}$ contribution depends only on the density, and not on the way this density is generated from the different components, the same $E_{e p p}$ is obtained if the density is generated entirely from one component only, e.g., by hypothesizing that this component of the wave function is the square root of the density, or whether this component is generated from the same square root multiplied by some fraction for one component, some other fraction for a second component, etc., or even by a less regular spread of the density over the components. However, one can hypothesize that in order to minimize the kinetic energy (or, equivalently, as a consequence of the quadratic behavior of the $H_{\mathrm{LK}}$ Hamiltonian with respect to the wave-vector length) the $E_{\mathrm{el}}$ will be minimal when all the components have the same spatial variations. Hence, one is lead to the following ansatz:

$$
\phi_{j}(\vec{r})=c_{j} \phi_{0}(\vec{r})
$$

where the coefficients $c_{j}$ are normalized,

$$
1=\sum_{j}\left|c_{j}\right|^{2}
$$

as well as the wave function $\phi_{0}(\vec{r})$ :

$$
1=\int d^{3} k \phi_{0}^{*}(\vec{k}) \phi_{0}(\vec{k})=\int d^{3} r \phi_{0}^{*}(\vec{r}) \phi_{0}(\vec{r})
$$

The density is written directly from $\phi_{0}(\vec{r})$ :

$$
\rho(\vec{r})=\left|\phi_{0}(\vec{r})\right|^{2} .
$$

In this case, the electronic energy becomes

$$
E_{\mathrm{el}}\left[\phi_{0}, c\right]=\left.\sigma \int d^{3} k \phi_{0}^{*}(\vec{k}) \phi_{0}(\vec{k}) \sum_{j, j^{\prime}=1}^{n} c_{j}^{*} H_{\mathrm{LK}}(\vec{k})\right|_{\left(j, j^{\prime}\right)} c_{j} .
$$

Moreover, $\left.\sum_{j, j^{\prime}=1}^{n} c_{j}^{*} H_{\mathrm{LK}}(\vec{k})\right|_{\left(j, j^{\prime}\right)} c_{j}$ is a quadratic form over the wave vectors, that has principal axes. Along these principal axes one recovers the usual dispersion relation

$$
E_{\text {kin }}(\vec{k})=\frac{k_{1}^{2}}{2 m_{1}^{*}}+\frac{k_{2}^{2}}{2 m_{2}^{*}}+\frac{k_{3}^{2}}{2 m_{3}^{*}},
$$

although the new 1,2, and 3 principal axes might not be aligned with the initially chosen 1,2 , and 3 states.

The search for the best trial wave function can proceed as follows.

(1) One chooses several possible $c_{j}$ sets.

(2) For each of these sets, one solves a nondegenerate optimization problem, of the kind already tackled in Sec. IV A, with effective masses $m_{n}^{*}$, for which the polaron formation energy can be computed as well as the localization length.

(3) Among the different $c_{j}$ sets, the best ansatz will be the one that delivers the lowest polaron formation energy, with corresponding localization length.

In practice, we consider for $c_{j}$ the three directions (100), (110), and (111), for which we know the effective masses [see Eq. (81)]. For the (100) and (111) directions, we recover the nondegenerate uniaxial case, as two of these effective masses are equal. However, for the (110) direction, the three effective masses are distinct, and the variational problem must be addressed entirely numerically, or using an approximate expression for $\left\langle a_{P d}^{-1}\right\rangle_{4 \pi}$, that we provide in the Supplemental Material [48].

\section{RESULTS}

We have applied our formalism to a range of (cubic) materials, including II-VI compounds CdS, CdSe, CdTe, ZnS, $\mathrm{ZnSe}$, and $\mathrm{ZnTe}$; III-V compounds AlAs, AlSb, AlP, BAs, $\mathrm{BN}, \mathrm{GaAs}, \mathrm{GaN}$, and $\mathrm{GaP}$; oxides $\mathrm{BaO}, \mathrm{CaO}, \mathrm{Li}_{2} \mathrm{O}, \mathrm{MgO}$, and $\mathrm{SrO}$; and $\mathrm{SiC}$ and $\mathrm{Cs}_{2} \mathrm{NaScF}_{6}$ (a case with multiple polar phonon modes) adding up to a total of 21 cases. In all the cases we study both hole and electrons polarons the characteristics of which are analyzed in the present section.

We find a range of polaron behaviors in the studied materials, among which we identified nine electron polarons in the nondegenerate and isotropic case, 11 electron polarons and one hole polaron in the nondegenerate and anisotropic case, and 20 hole polarons and one electron polaron in the threefold degenerate case.

In order to obtain parameters for the $\mathrm{gFr}$ model, we use density functional theory as implemented in the ABINIT package $[46,49,50]$. Calculation parameters (kinetic energy cutoff and reciprocal space sampling) for both the electronic and phononic wave vectors are provided in Table S1 in Supplemental Material [48]. The exchange-correlation energy has been treated using the generalized gradient approximation Perdew-Burke-Ernzerhof (GGA-PBE) approximation [51] with the corresponding norm-conserving pseudopotentials provided by the PseudoDojo project [52], except for $\mathrm{BN}-\mathrm{zb}$ where previously published results were used [22]. Although effective masses from GGA-PBE are not very accurate, it is sufficient to highlight the role of electronphonon coupling in electronic mass renormalization. Based on the converged electronic wave functions, density functional 


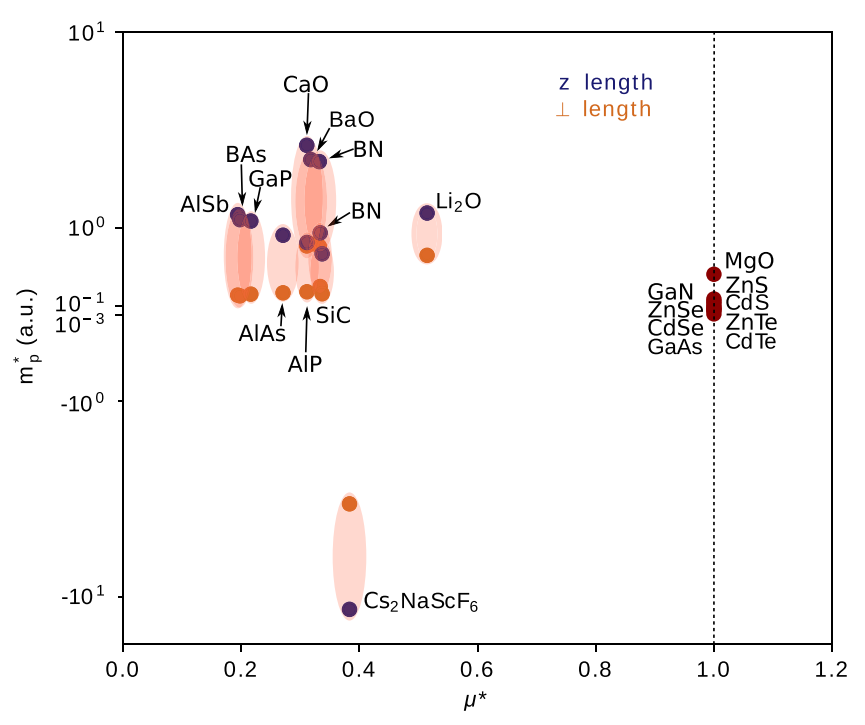

FIG. 4. Electron polaron effective mass as a function of the ratio between in-plane, $m_{\perp}$, and out-of-plane, $m_{z}$, bare electron effective masses for the studied materials. For the full set of values refer to Table S12 in Supplemental Material [48].

perturbation theory $[46,53]$ has been used in order to obtain the Luttinger parameters, electronic effective masses, dielectric constants, and $\Gamma$-point phonon frequencies. Parameters can be found in Tables S2 and S3 in Supplemental Material [48].

We show the corresponding electron and hole polaron effective masses in Figs. 4 and 5. For the nondegenerate case, this is presented as a function of the ratio between the inplane, $m_{\perp}$, and out-of-plane, $m_{z}$, electronic effective masses, referred to as $\mu^{*}$. The reason for which we depart from the spherical shape follows from the character of the electronic band structure. While for the spherical effective masses the conduction band minimum is at the Brillouin zone center, in the ellipsoidal case the minimum is found at $X$ or $L$. For the

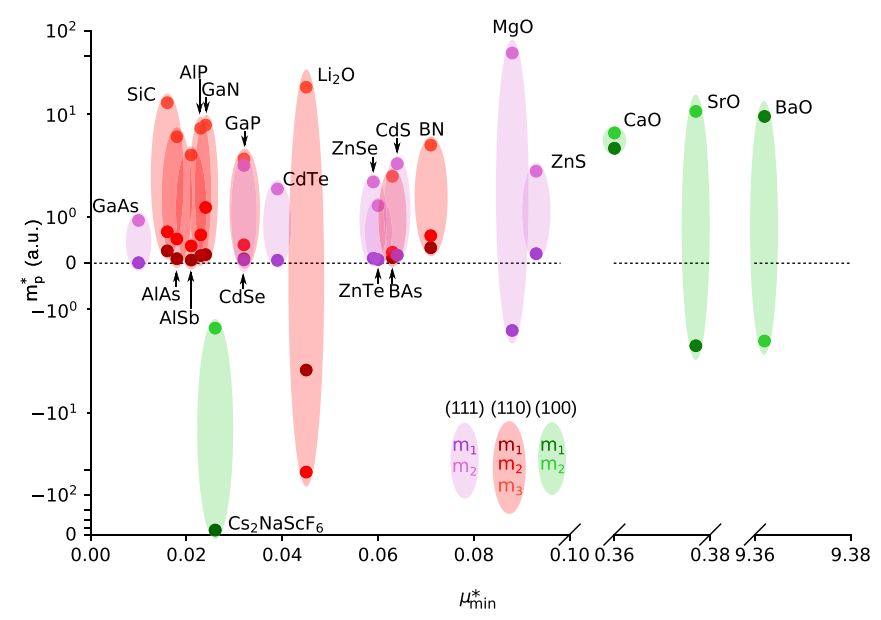

FIG. 5. Hole polaron effective mass as a function of the (minimum) ratio between the corresponding electronic effective masses for the studied materials. Negative values for the polaron effective masses indicate a crossing of the mass breakdown limit. For the full set of values refer to Table S6 in Supplemental Material [48].

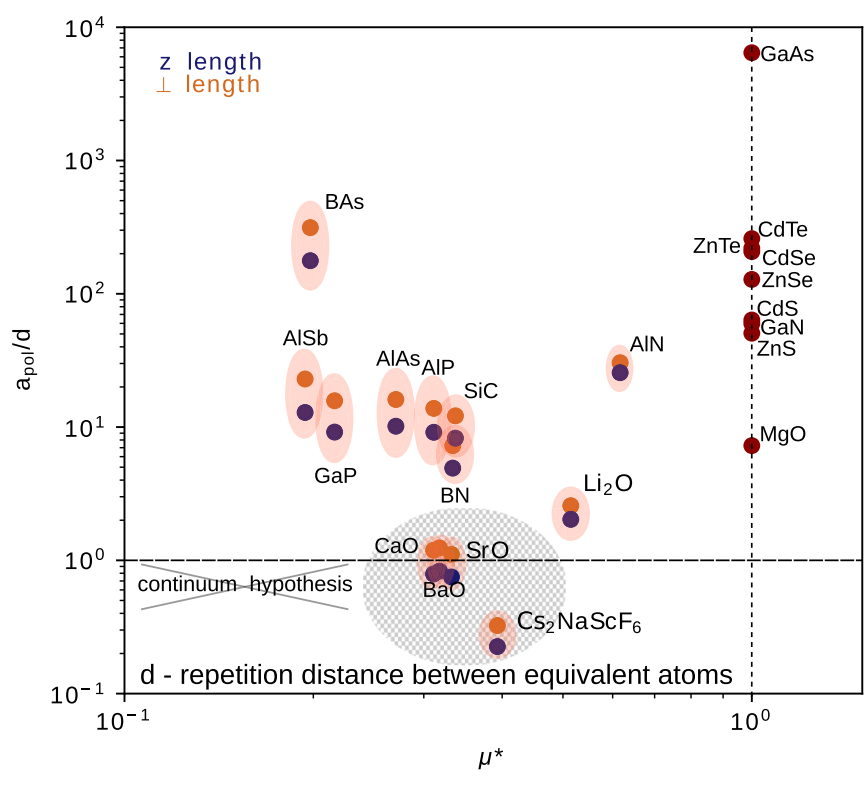

FIG. 6. Ratio between the electron polaron localization length and repetition distance $d$ between equivalent atoms as a function of the ratio between in-plane, $m_{\perp}$, and out-of-plane, $m_{z}$, effective masses for the studied materials. For the full set of values refer to Table S9 in Supplemental Material [48]. Note that when the two effective masses are equal, the isotropic case is reached and the characteristic localization length is isotropic (red dots), while for the other cases two localization lengths are necessary, for the in- and out-of-plane lengths (orange and blue dots).

degenerate case, we also use an anisotropy measure to plot the results, namely, the minimum ratio between the electronic effective masses of different bands, be it along the (100), the (110), or the (111) direction, depending on the direction along which the polaron has the lowest formation energy in the strong-coupling case. We note this quantity $\mu_{\min }^{*}$.

As a sanity check of the obtained values, for the degenerate case, we compute the sum of the three inverse polaron effective masses for different directions, and find that it is indeed constant, and independent of the direction in the Brillouin zone. This is expected based on the derivations above, from a similar property described in Eq. (81).

In Figs. 6 and 7 we report the localization length for the electron and hole polaron, respectively, in units of the crystal lattice constant. This is also presented as a function of the ratio between the in-plane, $m_{\perp}$, and out-of-plane, $m_{z}$, electronic effective masses, referred to as $\mu^{*}$. If $\mu^{*}=1$ we recover the spherically shaped polaron scenario, while for other values we describe ellipsoidal shaped polarons with two different axes: $a_{p_{\perp}}$ and $a_{p_{z}}$, respectively. More importantly, for some oxides the localization length is on the order of the distance between the atoms, which indicates a breakdown of the Fröhlich continuum hypothesis.

Along the (100) or (111) directions, polarons have a shape of an oblate spheroid for $\mu^{*}<1$, and a prolate one for $\mu^{*}>1$. The deviation from a sphere becomes more visible with increasing difference between the three radii characterizing the polaron, as we move away from $\mu^{*}=1$. In the case of (110) direction the degeneracy among the effective masses is lifted and the polaron takes the shape of a general ellipsoid. 


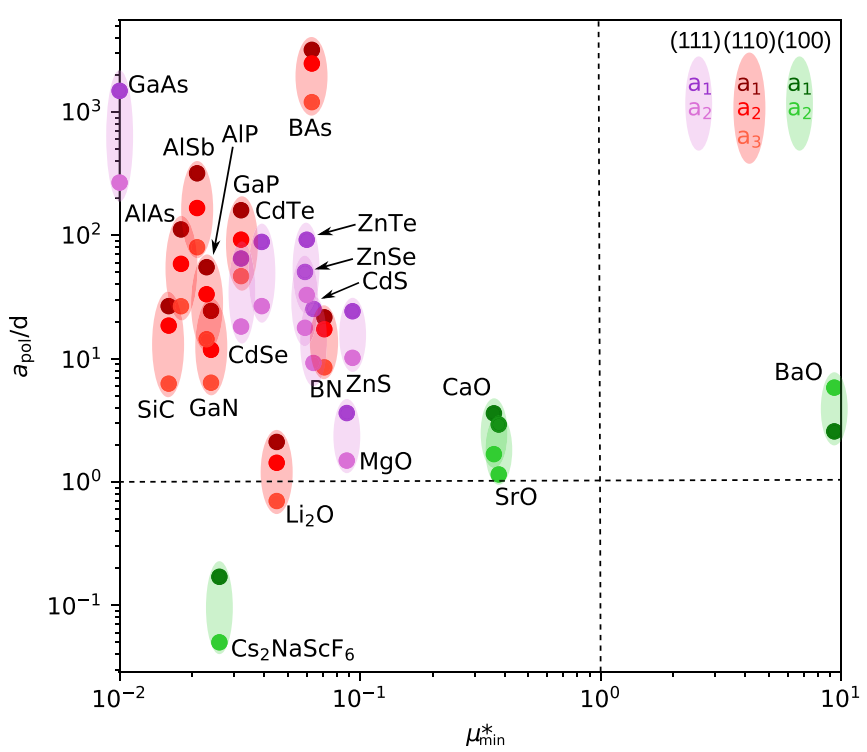

FIG. 7. Ratio between hole polaron localization lengths along the direction with lowest formation energy expressed and repetition distance between equivalent atoms as a function of the (lowest) ratio between the electronic effective masses for the studied materials. Note that in the case when the lowest polaron formation energy is along the (110) direction there is no degeneracy in the electronic effective masses and, consequently, the polaron has the shape of a triaxial ellipsoid. $a_{1}$ represents the polaron radius along the direction in which the polaron formation energy is the lowest, while $a_{2}$ (or $a_{3}$ ) represents that along the perpendicular direction.

We show in Fig. 8 the characteristics for both electron and hole polarons in the studied materials. The ratio between the electronic and polaronic effective masses serves as a reference relative to the strength of the electron-phonon coupling; thus

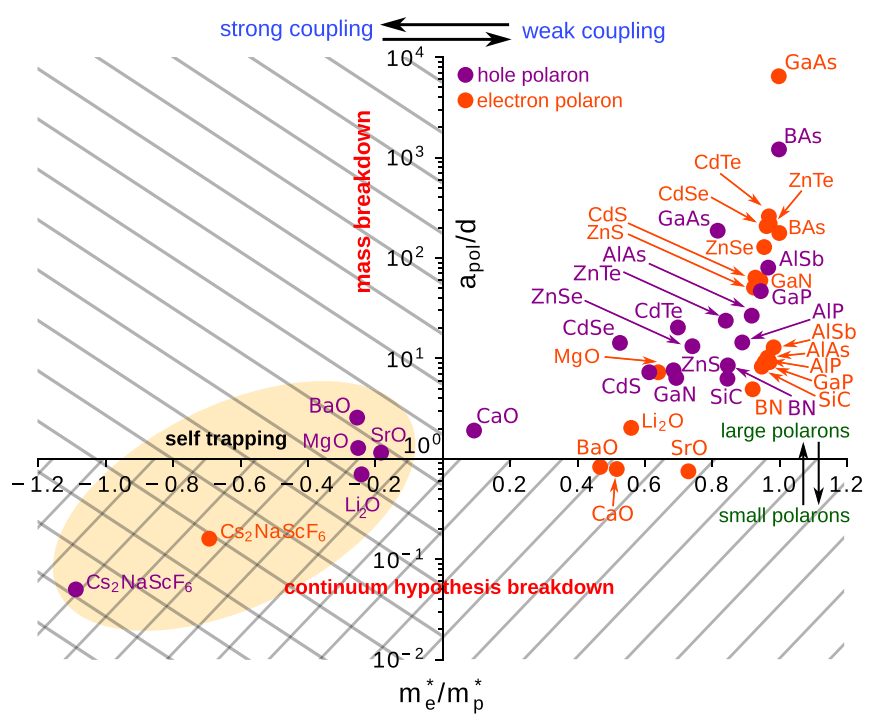

FIG. 8. Ratio between shortest electron and hole polaron localization lengths and repetition distance between equivalent atoms, as a function of the smallest ratio between electron (or hole) effective mass and polaron effective mass for the studied materials. When the latter drops below zero, perturbation theory breaks down, while when the former drops below 1 the continuum hypothesis breaks down. the closer the ratio is to 1 , the weaker the coupling. At the other end of the EPC effect we arrive at an opposite sign polaron effective mass with respect to the electronic one, going beyond the mass breakdown limit. This is a clear indication of self-trapping hole polaron formation in the indicated oxides. On the other hand, we set a relatively arbitrary breakdown $\left(a_{P} \approx d\right)$ to the continuum hypothesis inherent to the Fröhlich model $\left(a_{P} \gg d\right)$. In this case we show the shortest localization length in the cases where we do not find ourselves in the nondegenerate isotropic scenario. We note that for some of the studied materials the localization length is of the order of the repetition distance (e.g., $\mathrm{BaO}, \mathrm{SrO}, \mathrm{MgO}$, and $\mathrm{CdSe}$ ), and as a consequence we reach the continuum breakdown limit. In this case the discrete character of the crystal should be taken into account and another theoretical model might be more suitable for describing the polaron.

\section{DISCUSSION}

We show in Fig. 8 the characteristics (effective mass and localization length) for both electron and hole polarons in the studied materials. On the horizontal axis, the ratio between the electronic and polaronic effective masses characterizes the strength of the electron-phonon coupling: the closer the ratio is to 1 , the weaker the coupling, whereas for very strong EPC we arrive at a change of sign for the polaron effective mass. As previously discussed this is associated with a breakdown of weak-coupling perturbative treatment, and indicates that a strong-coupling approach is needed: self-trapped polarons will form in these oxides. On the other hand, we set a somewhat arbitrary breakdown threshold $\left(a_{P} \approx d\right)$ to characterize the applicability of the continuum hypothesis inherent to the Fröhlich model $\left(a_{P} \gg d\right)$. We show the shortest localization length in cases beyond the nondegenerate isotropic scenario.

We note that for some of the studied materials the localization length is of the order of the repetition distance (e.g., $\mathrm{BaO}$, $\mathrm{SrO}, \mathrm{CaO}, \mathrm{MgO}, \mathrm{Li}_{2} \mathrm{O}$, and $\mathrm{Cs}_{2} \mathrm{NaScF}_{6}$, for both electron and hole polarons, except the $\mathrm{MgO}$ electron polaron), and as a consequence we reach the continuum breakdown limit: the discrete character of the crystal should be taken into account, and another theoretical model would be more suitable to describe the polaron. The weak-coupling perturbation theory treatment also breaks down for the hole polarons of $\mathrm{BaO}, \mathrm{SrO}$, $\mathrm{MgO}$, and $\mathrm{Li}_{2} \mathrm{O}$. For all these cases, the ZPR obtained from perturbation theory (including the AHC approach) might be questioned.

The results from the Feynman approach, computed exactly for the isotropic mass situation, are averaged as described in Sec. II B. This is done for the nondegenerate anisotropic electronic dispersion.

In Fig. 9 we note that the variational approach reaches, as expected, a lower ground-state energy for both anisotropic and isotropic electronic dispersions. While in the isotropic case the relative difference between the two theoretical approaches remains within $2.5 \%$, the anisotropic case produces differences as high as $17.5 \%$ among the studied materials. Although in both treatments the wave function is constructed based on a Gaussian function, in the Feynman approach we consider an approximate treatment of the anisotropy which nevertheless yields a reasonable comparison. 


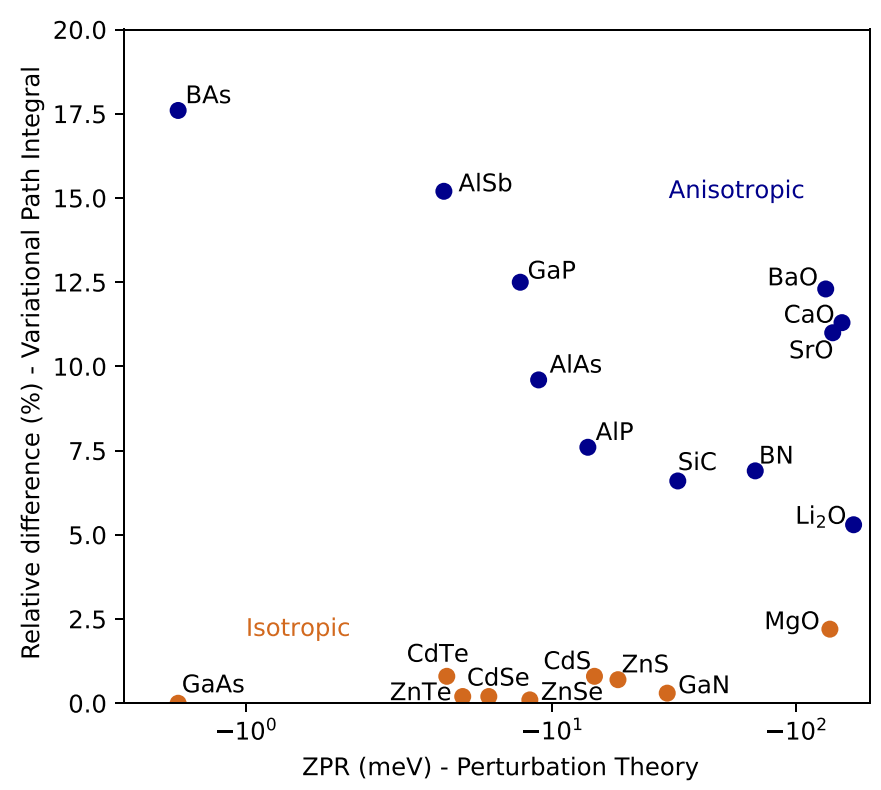

FIG. 9. In the nondegenerate electron polaron situation, relative difference between the ZPR determined using perturbation theory (fully taking into account the possible anisotropy) and Feynman variational path integral approach (with approximate treatment of the anisotropy). In all cases the ZPR determined using the perturbative method has a larger value than the ZPR determined using the variational approach. For the full set of values refer to Table S11 in Supplemental Material [48].

In Fig. 10 we present the relative difference in polaron effective mass between the above-mentioned theoretical approaches. We note that the largest difference is found in materials that, within the Fröhlich approach, are at the

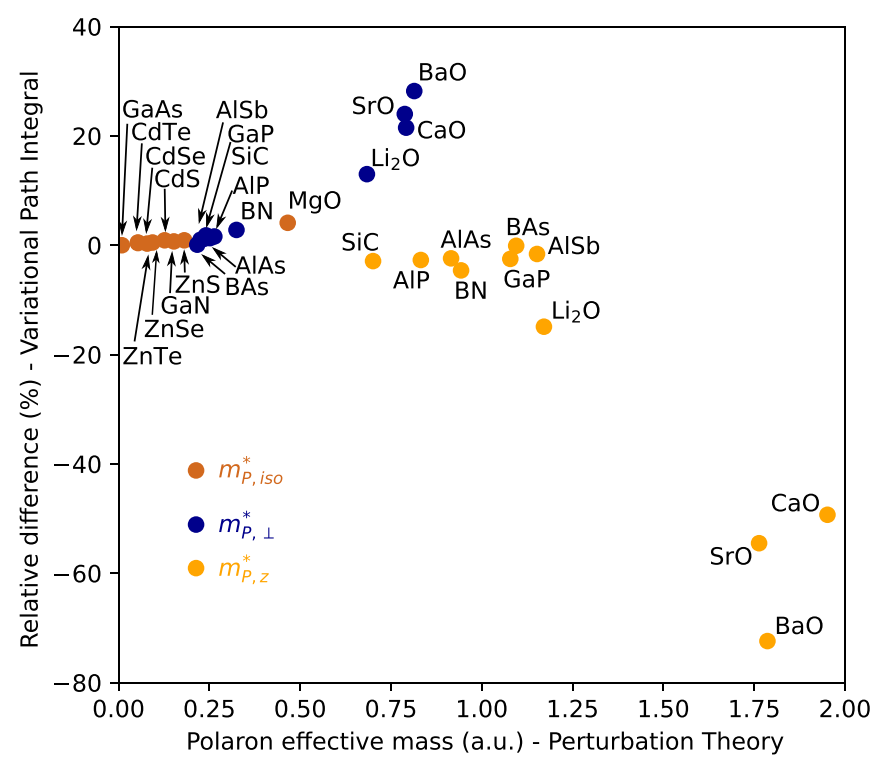

FIG. 10. In the nondegenerate electron polaron situation, relative difference between the effective masses determined using perturbation theory (fully taking into account the possible anisotropy) and Feynman variational path integral approach (with approximate treatment of the anisotropy). $m_{P, \text { iso }}^{*}$ is the isotropic effective mass, while in the anisotropic case $m_{P, \perp}^{*}$ and $m_{P, z}^{*}$ are the in-plane and out-of-plane polaron effective masses. continuum limit breakdown, like $\mathrm{BaO}, \mathrm{CaO}, \mathrm{SrO}$, and, to a lesser extent, $\mathrm{Li}_{2} \mathrm{O}$. In both anisotropic and isotropic cases we note that the relative difference increases with polaron effective mass, and the in-plane and out-of-plane effective mass differences seem to diverge. This sudden increase in the relative difference is associated with the lower $\alpha$ breakdown limit in the anisotropic case, as expressed in Fig. 1.

\section{CONCLUSIONS}

In this paper, we propose a formalism to describe realistic scenarios of polarons in cubic materials. Starting from the basic Fröhlich model, we include both anisotropic and degenerate electronic bands, and determine the basic characteristics of both electron and hole polarons (ground-state energy, localization lengths, and effective masses) for a set of 21 materials. We assess the limitations of the weak-coupling approach in determining the polaron effective masses: in lowest-order perturbation theory, several of the studied oxide compounds go beyond the mass breakdown limit. In generalizing the isotropic electronic energy dispersion, the mass breakdown limit is reached earlier depending on the degree of electronic anisotropy present in the system. We also perform strong-coupling calculations to determine the polaron's ground-state energy and localization length. We note that for the studied oxides we reach the continuum limit inherent to the Fröhlich model. We compare the perturbative treatment of the Fröhlich model for anisotropic electronic dispersions with the Feynman variational path integral approach, and find them to be in reasonable agreement in the weak-coupling regime. The Feynman path integral approach signals clearly where the perturbative approach fails to properly describe the polaronic behavior.

The main results of this paper are polaron properties for a set of 21 materials, obtained from the generalized Fröhlich model, with parameters determined from first principles. The increased range of applicability of the gFr model with respect to the standard Fröhlich model opens the possibility to analyze a wide spectrum of polarons, including the realistic treatment of effective mass anisotropy and possible band degeneracy.

\section{ACKNOWLEDGMENTS}

This work has been supported by the Fonds de la Recherche Scientifique (FRS-FNRS, Belgium) through PdR ALPS Grant No. T.0103.19, the Fonds de Recherche du Québec Nature et Technologie, and the Natural Sciences and Engineering Research Council of Canada under Grant No. RGPIN-2016-06666. Computational resources have been provided by the supercomputing facilities CISM of the Université Catholique de Louvain, the Consortium des Equipements de Calcul Intensif (CECI) en Fédération Wallonie Bruxelles funded by the FRS-FNRS under Grant No. 2.5020.11, the Canadian Foundation for Innovation, the Ministère de l'Éducation des Loisirs et du Sport (Québec), Calcul Québec, and Compute Canada. J.M.F. is supported by a Royal Society University Research Fellowship (Grant No. URF-R1191292). We thank Samuel Poncé for very useful comments on the paper. 
[1] C. Franchini, M. Reticcioli, M. Setvin, and U. Diebold, Nat. Rev. Mater. 6, 560 (2021).

[2] L. D. Landau, Phys. Z. Sowjetunion 3, 644 (1933).

[3] S. I. Pekar, Zh. Eksp. Teor. Fiz. 16, 341 (1946).

[4] L. D. Landau and S. I. Pekar, Zh. Eksp. Teor. Fiz. 18, 419 (1948).

[5] H. Fröhlich, H. Pelzer, and S. Zienau, Phil. Mag. 41, 221 (1950).

[6] R. P. Feynman, Phys. Rev. 97, 660 (1955).

[7] A. S. Mishchenko, N. V. Prokof'ev, A. Sakamoto, and B. V. Svistunov, Phys. Rev. B 62, 6317 (2000).

[8] J. Devreese and A. Alexandrov, Rep. Prog. Phys. 72, 066501 (2009).

[9] T. Holstein, Ann. Phys. (NY) 8, 325 (1959).

[10] T. Holstein, Ann. Phys. (NY) 8, 343 (1959).

[11] A. M. Stoneham, J. Gavartin, A. L. Shluger, A. V. Kimmel, D. Ramo, H. M. Rønnow, G. Aeppli, and C. Renner, J. Phys.: Condens. Matter 19, 255208 (2007).

[12] J. B. Varley, A. Janotti, C. Franchini, and C. G. Van de Walle, Phys. Rev. B 85, 081109(R) (2012).

[13] B. Sadigh, P. Erhart, and D. Aberg, Phys. Rev. B 92, 075202 (2015).

[14] G. Geneste, B. Amadon, M. Torrent, and G. Dezanneau, Phys. Rev. B 96, 134123 (2017).

[15] S. Kokott, S. V. Levchenko, P. Rinke, and M. Scheffler, New J. Phys. 20, 033023 (2018).

[16] A. Marini, Phys. Rev. Lett. 101, 106405 (2008).

[17] F. Giustino, S. G. Louie, and M. L. Cohen, Phys. Rev. Lett. 105, 265501 (2010).

[18] X. Gonze, P. Boulanger, and M. Côté, Ann. Phys. (Leipzig) 523, 168 (2011).

[19] S. Poncé, G. Antonius, P. Boulanger, E. Cannuccia, A. Marini, M. Côté, and X. Gonze, Comput. Mater. Sci. 83, 341 (2014).

[20] S. Poncé, G. Antonius, Y. Gillet, P. Boulanger, J. Laflamme Janssen, A. Marini, M. Côté, and X. Gonze, Phys. Rev. B 90, 214304 (2014).

[21] C. Verdi and F. Giustino, Phys. Rev. Lett. 115, 176401 (2015).

[22] S. Poncé, Y. Gillet, J. Laflamme Janssen, A. Marini, M. Verstraete, and X. Gonze, J. Chem. Phys. 143, 102813 (2015).

[23] F. Giustino, Rev. Mod. Phys. 89, 015003 (2017).

[24] J. P. Nery, P. B. Allen, G. Antonius, L. Reining, A. Miglio, and X. Gonze, Phys. Rev. B 97, 115145 (2018).

[25] W. H. Sio, C. Verdi, S. Poncé, and F. Giustino, Phys. Rev. Lett. 122, 246403 (2019).

[26] A. Miglio, V. Brousseau-Couture, E. Godbout, G. Antonius, Y.H. Chan, S. G. Louie, M. Côté, M. Giantomassi, and X. Gonze, npj Comput. Mater. 6, 167 (2020).

[27] F. Brown-Altvater, G. Antonius, T. Rangel, M. Giantomassi, C. Draxl, X. Gonze, S. G. Louie, and J. B. Neaton, Phys. Rev. B 101, 165102 (2020).
[28] N. A. Mecholsky, L. Resca, I. L. Pegg, and M. Fornari, Phys. Rev. B 89, 155131 (2014).

[29] J. T. Devreese, J. Phys.: Condens. Matter 19, 255201 (2007).

[30] J. T. Titantah, C. Pierleoni, and S. Ciuchi, Phys. Rev. Lett. 87, 206406 (2001).

[31] T. Hahn, S. Klimin, J. Tempere, J. T. Devreese, and C. Franchini, Phys. Rev. B 97, 134305 (2018).

[32] H.-R. Trebin and U. Rössler, Phys. Status Solidi B 70, 717 (1975).

[33] H. Fock, B. Kramer, and H. Büttner, Phys. Status Solidi B 67, 199 (1975).

[34] M. Schlipf, S. Poncé, and F. Giustino, Phys. Rev. Lett. 121, 086402 (2018).

[35] G. D. Mahan, J. Phys. Chem. Solids 26, 751 (1965).

[36] J. T. Devreese, S. N. Klimin, J. L. M. van Mechelen, and D. van der Marel, Phys. Rev. B 81, 125119 (2010).

[37] M. Houtput and J. Tempere, Phys. Rev. B 103, 184306 (2021).

[38] H. Fröhlich, Proc. R. Soc. A 215, 291 (1952).

[39] J. P. Nery and P. B. Allen, Phys. Rev. B 94, 115135 (2016).

[40] G. D. Mahan, Many-Particle Physics (Kluwer, New York, 2000).

[41] S. J. Miyake, J. Phys. Soc. Jpn. 41, 747 (1976).

[42] Y. Ōsaka, Prog. Theor. Phys. 22, 437 (1959).

[43] R. P. Feynman, R. W. Hellwarth, C. K. Iddings, and P. M. Platzman, Phys. Rev. 127, 1004 (1962).

[44] F. Peeters and J. Devreese, in Theory of Polaron Mobility (Academic Press, New York, 1984), pp. 81-133.

[45] T. D. Schultz, Phys. Rev. 116, 526 (1959).

[46] X. Gonze and C. Lee, Phys. Rev. B 55, 10355 (1997).

[47] J. M. Luttinger and W. Kohn, Phys. Rev. 97, 869 (1955).

[48] See Supplemental Material at http://link.aps.org/supplemental/ 10.1103/PhysRevB.104.235123 for the data to reproduce the figures and the theoretical limits of the uniaxial and ellipsoidal cases.

[49] X. Gonze, B. Amadon, G. Antonius, F. Arnardi, L. Baguet, J.M. Beuken, J. Bieder, F. Bottin, J. Bouchet, E. Bousquet, N. Brouwer, F. Bruneval, G. Brunin, T. Cavignac, J.-B. Charraud, W. Chen, M. Côté, S. Cottenier, J. Denier, G. Geneste et al., Comput. Phys. Commun. 248, 107042 (2020).

[50] X. Gonze, F. Jollet, F. Abreu Araujo, D. Adams, B. Amadon, T. Applencourt, C. Audouze, J.-M. Beuken, J. Bieder, A. Bokhanchuk, E. Bousquet, F. Bruneval, D. Caliste, M. Côté, F. Dahm, F. Da Pieve, M. Delaveau, M. Di Gennaro, B. Dorado, C. Espejo et al., Comput. Phys. Commun. 205, 106 (2016).

[51] J. P. Perdew, K. Burke, and M. Ernzerhof, Phys. Rev. Lett. 77, 3865 (1996).

[52] M. van Setten, M. Giantomassi, E. Bousquet, M. Verstraete, D. Hamann, X. Gonze, and G.-M. Rignanese, Comput. Phys. Commun. 226, 39 (2018).

[53] J. Laflamme Janssen, Y. Gillet, S. Poncé, A. Martin, M. Torrent, and X. Gonze, Phys. Rev. B 93, 205147 (2016). 